\title{
Parameter Permutation Symmetry in Particle Systems and Random Polymers
}

\author{
Leonid PETROV ab
}

a) University of Virginia, Department of Mathematics, 141 Cabell Drive, Kerchof Hall, P.O. Box 400137, Charlottesville, VA 22904, USA E-mail: lenia.petrov@gmail.com

URL: http://lpetrov.cc

b) Institute for Information Transmission Problems, Bolshoy Karetny per. 19, Moscow, 127994, Russia

Received October 26, 2020, in final form February 20, 2021; Published online March 06, 2021 https://doi.org/10.3842/SIGMA.2021.021

\begin{abstract}
Many integrable stochastic particle systems in one space dimension (such as TASEP - totally asymmetric simple exclusion process - and its various deformations, with a notable exception of ASEP) remain integrable when we equip each particle $x_{i}$ with its own jump rate parameter $\nu_{i}$. It is a consequence of integrability that the distribution of each particle $x_{n}(t)$ in a system started from the step initial configuration depends on the parameters $\nu_{j}, j \leq n$, in a symmetric way. A transposition $\nu_{n} \leftrightarrow \nu_{n+1}$ of the parameters thus affects only the distribution of $x_{n}(t)$. For $q$-Hahn TASEP and its degenerations ( $q$-TASEP and directed beta polymer) we realize the transposition $\nu_{n} \leftrightarrow \nu_{n+1}$ as an explicit Markov swap operator acting on the single particle $x_{n}(t)$. For beta polymer, the swap operator can be interpreted as a simple modification of the lattice on which the polymer is considered. Our main tools are Markov duality and contour integral formulas for joint moments. In particular, our constructions lead to a continuous time Markov process $Q^{(t)}$ preserving the time $\mathrm{t}$ distribution of the $q$-TASEP (with step initial configuration, where $t \in \mathbb{R}_{>0}$ is fixed). The dual system is a certain transient modification of the stochastic $q$-Boson system. We identify asymptotic survival probabilities of this transient process with $q$-moments of the $q$-TASEP, and use this to show the convergence of the process $\mathrm{Q}^{(\mathrm{t})}$ with arbitrary initial data to its stationary distribution. Setting $q=0$, we recover the results about the usual TASEP established recently in [arXiv:1907.09155] by a different approach based on Gibbs ensembles of interlacing particles in two dimensions.
\end{abstract}

Key words: $q$-TASEP; stochastic $q$-Boson system; stationary distribution; coordinate Bethe ansatz; $q$-Hahn TASEP

2020 Mathematics Subject Classification: 82C22; 60C05; 60J27

\section{Introduction}

\subsection{Overview}

In the past two decades, integrable stochastic interacting particle systems in one space dimension have been crucial in explicitly describing new universal asymptotic phenomena, most notably those corresponding to the Kardar-Parisi-Zhang universality class [19, 21, 31, 43]. By integrability in a stochastic system we mean the presence of exact formulas for probability distributions for a wide class of observables. Asymptotic (long time and large space) behavior of the system can be recovered by an analysis of these formulas. Initial successes with integrable stochastic particle systems were achieved through the use of determinantal point process techniques, e.g., see [34] for the asymptotic fluctuations of TASEP (totally asymmetric simple exclusion process). 
More recently new tools borrowed from quantum integrability, Bethe ansatz, and/or symmetric functions were applied to deformations of TASEP and related models:

- ASEP, in which particles can jump in both directions, but with different $\operatorname{rates}^{1}[50,51]$;

- random polymers such as the semi-discrete directed Brownian polymer [37], log-gamma polymer [23, 38, 47], or beta type polymers [4, 17, 22, 36, 49];

- $q$-TASEP and $q$-Hahn TASEP, in which particles jump in one direction, but with $q$-deformed jump rates $[7,10,20,27,42]$.

All these and several other integrable models can be unified under the umbrella of stochastic vertex models $[8,13,16,24]$.

Ever since the original works on TASEP around the year 2000 it was clear [29, 32] that integrability of some particle systems like TASEP is preserved in the presence of countably many extra parameters, for example, when each particle is equipped with its own jump rate. We will refer to such more general systems as multiparameter ones. This notion should be contrasted with the $q$-deformation by means of just one extra parameter which takes TASEP to $q$-TASEP. The latter is much more subtle and relies on passing to a deformed algebraic structure - for the $q$-TASEP, one replaces the Schur symmetric functions with the $q$-Whittaker ones.

It should be noted that TASEP in inhomogeneous space (when the jump rate of a particle depends on its location) does not seem to be integrable $[25,33,46]$ (cf. recent asymptotic fluctuation results $[5,6]$ requiring very delicate asymptotic analysis). Moreover, it is not known whether ASEP has any integrable multiparameter deformations. The stochastic six vertex model $[8,30]$ scales to ASEP and admits such a multiparameter deformation [14], but this deformation is destroyed by the scaling. Recently other families of spatially inhomogeneous integrable stochastic particle systems in one and two space dimensions were studied in [1, 15, 35, 40].

All known multiparameter integrable stochastic particle systems display a common feature. Namely, certain joint distributions in these systems are symmetric under (suitably restricted classes of) permutations of the parameters. This symmetry is far from being evident from the beginning, and is often observed only as a consequence of explicit formulas. The main goal of the present paper is to explore probabilistic consequences of parameter symmetries in integrable particle systems.

Recently a number of other papers investigating symmetries of multiparameter integrable stochastic particle systems and vertex models have appeared [12, 18, 26, 28]. So far it is not clear whether those results have any direct connection to the results of the present paper.

\section{$1.2 \quad$ Distributional symmetry of the $q$-Hahn TASEP}

The most general system we consider is the $q$-Hahn TASEP started from the step initial configuration $x_{n}(0)=-n, n=1,2, \ldots$. That is, every site of $\mathbb{Z}_{<0}$ is occupied by a particle, and every site of $\mathbb{Z}_{\geq 0}$ is empty. Throughout the paper we denote this configuration by step for short.

The $q$-Hahn TASEP was introduced in [42] and studied in [9, 20, 52]. Its multiparameter deformation appears in [14]. Under this deformation, each particle $x_{n}$ carries its own parameter $\nu_{n} \in(0,1)$ which determines the jump distribution of the particle. The $q$-Hahn TASEP is a discrete time Markov process on particle configurations in $\mathbb{Z}$. At each time step, every particle $x_{i}$ independently jumps to the right by $j$ steps with probability

$$
\varphi_{q, \gamma \nu_{i}, \nu_{i}}\left(j \mid x_{i-1}-x_{i}-1\right), \quad j \in\left\{0,1, \ldots, x_{i-1}-x_{i}-1\right\},
$$

\footnotetext{
${ }^{1}$ We say that an event in continuous time happens at rate $\alpha$ if $\mathbb{P}($ waiting time till the event occurs $>t)=\mathrm{e}^{-\alpha t}$ for all $t \in \mathbb{R}_{\geq 0}$.
} 
where $x_{0}=+\infty$, by agreement. Here $\varphi$ is the $q$-deformed beta-binomial distribution (Definition 3.1). See Fig. 1 for an illustration.

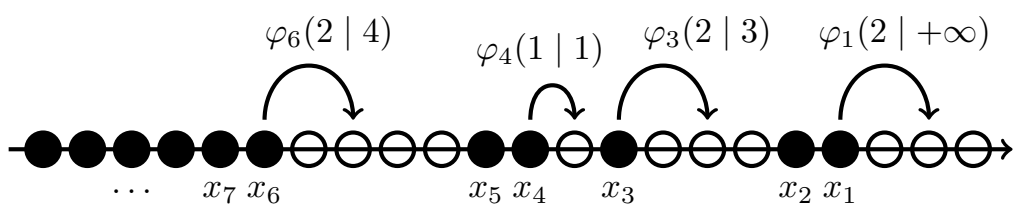

Figure 1. An example of a one-step transition in the $q$-Hahn TASEP, together with the corresponding probabilities for each particle. Here $\varphi_{i} \equiv \varphi_{q, \gamma \nu_{i}, \nu_{i}}$.

The distribution of each particle $x_{n}(t)$ at any time moment $t \in \mathbb{Z}_{\geq 0}$ in the $q$-Hahn TASEP started from step depends on the parameters $\nu_{1}, \ldots, \nu_{n}$ in a symmetric way. We check this symmetry using exact formulas in Section 3.2. The main structural result of the present paper is

Theorem 1.1 (Theorem 3.8 in the text). The elementary transposition $\nu_{n} \leftrightarrow \nu_{n+1}, \nu_{n+1}<\nu_{n}$, of two neighboring parameters in the q-Hahn TASEP started from step is equivalent in distribution to the action of an explicit Markov swap operator $p_{n}^{\mathrm{qH}}$ on the particle $x_{n}$. This operator moves $x_{n}$ to a random new location $x_{n}^{\prime}$ chosen with probability

$$
\varphi_{q, \frac{\nu_{n+1}}{\nu_{n}}, \nu_{n+1}}\left(x_{n}^{\prime}-x_{n+1}-1 \mid x_{n}-x_{n+1}-1\right), \quad x_{n}^{\prime} \in\left\{x_{n+1}+1, \ldots, x_{n}-1, x_{n}\right\},
$$

where $\varphi$ is the q-deformed beta-binomial distribution (Definition 3.1). The equivalence in distribution holds at any fixed time $t \in \mathbb{Z}_{\geq 0}$ in the q-Hahn TASEP, while the swap operator $p_{n}^{\mathrm{qH}}$ does not depend on $t$. See Fig. 2 for an illustration.

We prove this result in Section 3.3 using $q$-moment contour integral formulas and duality results $^{2}$ for the $q$-Hahn TASEP. Let us make a couple of remarks on the generality of the result and our methods.
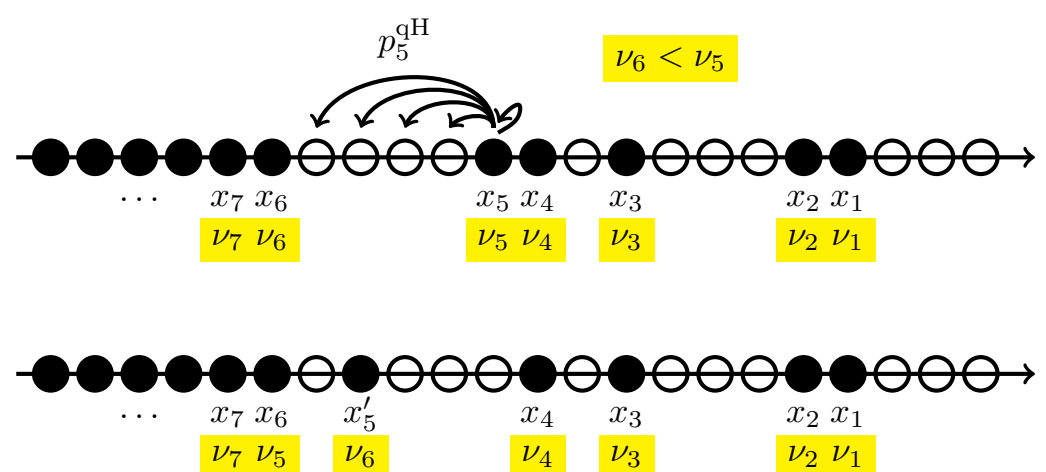

Figure 2. An example of the swap operator $p_{5}^{\mathrm{qH}}$ acting on the fifth particle in the $q$-Hahn TASEP (at an arbitrary time $t \in \mathbb{Z}_{\geq 0}$ ). Arrows show possible new locations of $x_{5}$ (note that with some probability it can stay in the same location). The resulting configuration (below) is distributed as the $q$-Hahn TASEP at the same time, but with the swapped parameters $\nu_{5} \leftrightarrow \nu_{6}$. The distributional identity holds only if $\nu_{6}<\nu_{5}$ before the swap.

First, note that there are certain other classes of initial data (for example, half-stationary) for which the $q$-Hahn TASEP displays parameter symmetry. Moreover, via the spectral theory of [9] one sees that for fairly general initial data the swap operators simultaneously applied to

\footnotetext{
${ }^{2}$ Sometimes, especially in the context of random polymers, this set of tools is referred to as "rigorous replica method".
} 
the particle system and the initial distribution lead to permutations of the parameters as in Theorem 1.1. For simplicity, in this paper we focus only on the step initial configuration.

Second, we expect that under suitable modifications the results of the present paper could carry over to the stochastic six vertex model [8] and the higher spin stochastic six vertex model $[14,24]$. However, as duality relations for these vertex models are more involved than the ones for the $q$-Hahn TASEP, it is not immediately clear how to extend the methods of the present paper to the vertex models. Therefore, we restrict out attention here to the $q$-Hahn TASEP.

\subsection{Applications}

We explore a number of interesting consequences of the distributional symmetry of the $q$-Hahn TASEP realized by the swap operators. Let us briefly describe them.

We take a continuous time limit of the $q$-Hahn TASEP and the swap operators. Denote by $\mathcal{M}_{q, \nu ; \mathrm{t}}^{\mathrm{qH}}$ the distribution of the parameter homogeneous (i.e., $\nu_{n} \equiv \nu$ ), continuous time $q$-Hahn TASEP at time $t \in \mathbb{R}_{\geq 0}$ started from step (see Section 4.2 for a detailed definition). The $q$-Hahn TASEP evolution acts on $\mathcal{M}_{q, \nu ; \mathrm{t}}^{\mathrm{qH}}$ by increasing the time parameter $\mathrm{t}$. We find that a suitable continuous limit as $r \rightarrow 1$ of the swap operators with $\nu_{n}=\nu r^{n-1}$ produces a (time-inhomogeneous) continuous time Markov process $\mathcal{B}^{\mathrm{qH}}$ on particle configurations. Starting from a random particle configuration distributed as $\mathcal{M}_{q, \nu ; \mathrm{t}}^{\mathrm{qH}}$ and running the process $\mathcal{B}^{\mathrm{qH}}$ for time $\tau \geq 0$, we get a configuration distributed as $\mathcal{M}_{q, \nu \mathrm{e}^{-\tau} ; \mathrm{te}^{-\tau}}^{\mathrm{qH}}$, that is, in which both parameters $\nu$ and $\mathrm{t}$ are rescaled. See Theorem 4.7 for a detailed formulation and Fig. 6 for an illustration of the two actions. When $\nu=0$, the backward process becomes time-homogeneous, and we discuss this case in more detail in the next Section 1.4.

When $q=\nu=0$, Theorem 4.7 recovers one of the main results of the recent work [41] on the existence of a time-homogeneous, continuous time process mapping the distributions of the usual TASEP back in time. We remark that the proof of this result following from the present paper is completely different from the argument given in [41]. The latter went through the well-known connection of the TASEP distribution and a Schur process [39] on interlacing arrays (about this connection see, e.g., [11]). For Schur processes, the two-dimensional version of the swap operator is accessible by elementary means.

In a scaling limit $q, \nu_{n} \rightarrow 1$, the $q$-Hahn TASEP turns into the beta polymer model introduced in [4]. In Section 6 we construct swap operators for the multiparameter version of the beta polymer model. The argument is formally independent of the rest of the paper, but proceeds through the same steps. For polymers, the swap operator can be realized as a simple modification of the lattice on which the beta polymer is defined. See Theorem 6.4 for a detailed formulation of the result, and Figs. 10 and 11 for illustrations of lattice modifications.

\subsection{Stationary dynamics on the $q$-TASEP distribution}

The last application concerns $q$-TASEP $[7,10]$, which is a $\nu=0$ degeneration of the $q$-Hahn TASEP. Let us focus on this case in more detail. Under the $q$-TASEP, each particle $x_{n}$ jumps to the right by one in continuous time at rate $1-q^{x_{n-1}-x_{n}-1}$, where $x_{0}=+\infty$, by agreement. In particular, we take the homogeneous $q$-TASEP in which all particles behave in the same manner. Denote by $\mathcal{M}_{q ; \mathrm{t}}^{\mathrm{qT}}$ the time $\mathrm{t}$ distribution of this continuous time $q$-TASEP started from the step initial configuration step.

When $\nu=0$, Theorem 4.7 produces a new time-homogeneous, continuous time Markov process which we denote by $\mathrm{Q}^{(\mathrm{t})}$, with the following properties:

- The process $\mathrm{Q}^{(\mathrm{t})}$ is a combination of two independent dynamics: the $q$-TASEP evolution, and the (slowed down by the factor of $\mathrm{t}$ ) backward $q$-TASEP evolution. The latter is 
a suitable degeneration of the backward $q$-Hahn process $\mathcal{B}^{\mathrm{qH}}$. Under this degeneration, the backward process becomes time-homogeneous. See Section 5.2 for the full definition of the process $\mathrm{Q}^{(\mathrm{t})}$.

- (Proposition 5.3) The process $\mathrm{Q}^{(\mathrm{t})}$ preserves the distribution $\mathcal{M}_{q ; \mathrm{t}}^{\mathrm{qT}}$. Here the time parameter $\mathrm{t} \in \mathbb{R}_{\geq 0}$ of the $q$-TASEP distribution $\mathcal{M}_{q ; \mathrm{t}}^{\mathrm{qT}}$ is fixed and is incorporated into the definition of the stationary process $\mathrm{Q}^{(\mathrm{t})}$.

- (Theorem 5.7) Start the process $\mathrm{Q}^{(\mathrm{t})}$ from an arbitrary particle configuration on $\mathbb{Z}$ which is empty far to the right, densely packed far to the left, and is balanced (in the sense that the number of holes to the left of zero equals the number of particles to the right of zero). Then in the long time limit the distribution of this process converges to the $q$-TASEP distribution $\mathcal{M}_{q ; \mathrm{t}}^{\mathrm{qT}}$.

We establish Theorem 5.7 by making use of duality for the stationary process $\mathrm{Q}^{(\mathrm{t})}$ which extends the duality between the $q$-TASEP and the stochastic $q$-Boson process from [10] (the stochastic $q$-Boson process dates back to [45]). In fact, we are able to use the same duality functional (corresponding to joint $q$-moments) for $\mathrm{Q}^{(\mathrm{t})}$. As a result we find that the process dual to $\mathrm{Q}^{(\mathrm{t})}$ is a new transient modification of the stochastic $q$-Boson process. The long time limit of this transient process is readily accessible, and Theorem 5.7 follows by matching the long time behavior of all $q$-moments of the stationary dynamics $\mathrm{Q}^{(\mathrm{t})}$ (with an arbitrary initial configuration) with those of the $q$-TASEP (with the step initial configuration).

Let us illustrate the transient modification in the simplest case of the first $q$-moment. Consider the continuous time random walk $n(\mathrm{t})$ on $\mathbb{Z}_{\geq 0}$ which jumps from $k$ to $k-1, k \geq 1$, at rate $1-q$. When the walk reaches zero, it stops. From the $q$-TASEP duality [10] we have

$$
\mathbb{E}_{\text {step }}^{\mathrm{qT}} q^{x_{n}(\mathrm{t})+n}=\mathbb{P}(n(\mathrm{t})>0 \mid n(0)=n), \quad n=1,2, \ldots
$$

Here the left-hand side is the expectation over the $q$-TASEP distribution $\mathcal{M}_{q ; \mathrm{t}}^{\mathrm{qT}}$, and the righthand side corresponds to the random walk $n(\mathrm{t})$. Similarly to (1.1), joint $q$-moments of the $q$-TASEP are governed by a multiparticle version of the process $n(\mathrm{t})$ - the stochastic $q$-Boson system.

Let us now fix the $q$-TASEP time parameter $t \in \mathbb{R}_{>0}$, and consider a different random walk $n^{(\mathrm{t})}(\tau)$ on $\mathbb{Z}_{\geq 0}$ (here $\tau$ is the new continuous time variable) with the following jump rates:

$$
\operatorname{rate}(k \rightarrow k-1)=1-q, \quad \operatorname{rate}(k-1 \rightarrow k)=\frac{k-1}{\mathrm{t}}, \quad k=1,2, \ldots
$$

This process has a single absorbing state 0 , and otherwise is transient. In other words, after a large time $\tau$, the particle $n^{(\mathrm{t})}(\tau)$ is either at 0 , or runs off to infinity. Note however that this process does not make infinitely many jumps in finite time. The duality for the stationary process $Q^{(t)}$ which we prove in this paper states (in the simplest case) that

$$
\mathbb{E}_{\text {step }}^{\text {stat }(\mathrm{t})} q^{x_{n}(\tau)+n}=\mathbb{P}\left(n^{(\mathrm{t})}(\tau)>0 \mid n^{(\mathrm{t})}(0)=n\right), \quad n=1,2, \ldots
$$

Here the left-hand side is the expectation over the stationary process started from step, and the right-hand side may be called the survival probability (up to time $\tau$ ) of the transient random walk $n^{(\mathrm{t})}$. See Corollary 5.6 for the general statement which connects joint $q$-moments of the stationary process $\mathrm{Q}^{(\mathrm{t})}$ with a multiparticle version of $n^{(\mathrm{t})}(\tau)$. We call this multiparticle process the transient stochastic q-Boson system.

Taking the long time limit of (1.2), we see that

$$
\lim _{\tau \rightarrow+\infty} \mathbb{E}_{\text {step }}^{\text {stat }(\mathrm{t})} q^{x_{n}(\tau)+n}=\mathbb{P}\left(\lim _{\tau \rightarrow+\infty} n^{(\mathrm{t})}(\tau)=+\infty \mid n^{(\mathrm{t})}(0)=n\right), \quad n=1,2, \ldots
$$


The right-hand side is the asymptotic survival probability that the transient random walk eventually runs off to infinity and is not absorbed at zero. This probability, viewed as a function of the initial location $n$, is a harmonic function ${ }^{3}$ for the transient random walk $n^{(\mathrm{t})}(\tau)$, which, moreover, takes value 1 at $n=+\infty$. This identifies the harmonic function uniquely. From the stationarity of $\mathcal{M}_{q ; \mathrm{t}}^{\mathrm{q}}$ under the process $\mathrm{Q}^{(\mathrm{t})}$ one can check that (1.1) satisfies the same harmonicity condition, which implies that (1.3) equals (1.1).

A general multiparticle argument involves identifying the "correct" harmonic function (asymptotic survival probability) of the transient stochastic $q$-Boson system with the joint $q$-moments of the $q$-TASEP. This identification requires additional technical steps since the space of harmonic functions for the multiparticle process is higher-dimensional. Along this route we obtain the proof that $\mathrm{Q}^{(\mathrm{t})}$ converges to its stationary distribution $\mathcal{M}_{q ; \mathrm{t}}^{\mathrm{qT}}$ (Theorem 5.7).

\subsection{Outline}

In Section 2 we give general definitions related to parameter-symmetric particle systems and swap operators. In Section 3 for the $q$-Hahn TASEP we present an explicit realization of the parameter transposition in terms of a Markov swap operator corresponding to a random jump of a single particle. In Section 4 we pass to the continuous time in the $q$-Hahn TASEP, and obtain the $q$-Hahn backward process. This also implies the results about the TASEP from [41]. In Section 5 we define and study the dynamics preserving the $q$-TASEP distribution, and show its convergence to stationarity. In Section 6 we obtain swap operators for the beta polymer.

\section{From symmetry to swap operators}

This section contains an abstract discussion of stochastic particle systems on $\mathbb{Z}$ which depend symmetrically on their parameters. The main notions which we use in other parts of the paper are parameter-symmetric stochastic particle system and swap operators.

\subsection{Parameter-symmetric particle systems}

Let $\operatorname{Conf}_{\text {fin }}(\mathbb{Z})$ be the space of particle configurations $\mathbf{x}=\left(\cdots<x_{3}<x_{2}<x_{1}\right), x_{i} \in \mathbb{Z}$, which can be obtained from the step configuration step $:=(\ldots,-3,-2,-1)$ by finitely many operations of moving a particle to the right by one into the nearby empty spot. The space $\operatorname{Conf}_{\text {fin }}(\mathbb{Z})$ is countable.

By a multiparameter interacting particle system $\mathbf{x}(t)$ we mean a Markov process on $\operatorname{Conf}_{\text {fin }}(\mathbb{Z})$ evolving in continuous or discrete time, such that $\mathbf{x}(0)=$ step. Assume that this Markov process depends on countably many parameters $\boldsymbol{\nu}=\left\{\nu_{i}\right\}_{i \in \mathbb{Z}_{>1}}$. The parameters $\nu_{i}$ in our situation are real, though without loss of generality they may belong to an abstract space. One should think that $\nu_{i}$ is attached to the particle $x_{i}$, but the distribution of each $x_{j}(t)$ may depend on all of the $\nu_{i}$ 's. We denote the process depending on $\boldsymbol{\nu}$ by $\mathbf{x}^{\boldsymbol{\nu}}(t)$. In this section we assume that all the parameters $\nu_{i}$ are pairwise distinct.

The infinite symmetric group $S(\infty)=\bigcup_{n=1}^{\infty} S(n)$ acts on the parameters $\boldsymbol{\nu}$ by permutations, $\sigma: \boldsymbol{\nu} \mapsto \sigma \boldsymbol{\nu}$. Here $S(n)$ is the symmetric group which permutes only the first parameters $\nu_{1}, \ldots, \nu_{n}$. Let us denote by $S_{n}(\infty) \subset S(\infty)$ the subgroup which permutes $\nu_{n+1}, \nu_{n+2}, \ldots$ and maps each $\nu_{i}, 1 \leq i \leq n$, into itself. Note that $S(n) \cap S_{n}(\infty)=S(n) \cap S_{n-1}(\infty)=\{e\}$, and $S(n+1) \cap S_{n-1}(\infty)=\left\{e, s_{n}\right\}$, where $e$ is the identity permutation, and $s_{n}=(n, n+1)$ is the transposition $n \leftrightarrow n+1$.

\footnotetext{
${ }^{3} \mathrm{~A}$ harmonic function for a continuous time Markov process on a discrete space is a function which is eliminated by the infinitesimal generator of the process.
} 
By imposing a specific distributional symmetry of $\mathbf{x}^{\boldsymbol{\nu}}(t)$ under the action of $S(\infty)$ on the parameters $\boldsymbol{\nu}$, we arrive at the following definition:

Definition 2.1. A multiparameter particle system $\mathbf{x}^{\boldsymbol{\nu}}(t)$ is called parameter-symmetric, if for all $n$ and $t$ we have the following equality of joint distributions:

$$
\begin{aligned}
& \left(\ldots, x_{n+2}^{\nu}(t), x_{n+1}^{\nu}(t), x_{n-1}^{\nu}(t), \ldots, x_{1}^{\nu}(t)\right) \\
& \quad \stackrel{d}{=}\left(\ldots, x_{n+2}^{s_{n} \nu}(t), x_{n+1}^{s_{n} \nu}(t), x_{n-1}^{s_{n} \nu}(t), \ldots, x_{1}^{s_{n} \nu}(t)\right) .
\end{aligned}
$$

That is, the transposition $s_{n}$ preserves the joint distribution of all particles except $x_{n}$.

Here is a straightforward corollary of this definition:

Corollary 2.2. In a parameter-symmetric particle system, for any $t$ and any $\sigma \in S(n) \cup S_{n}(\infty)$, the random variables $x_{n}^{\nu}(t)$ and $x_{n}^{\sigma \nu}(t)$ have the same distribution.

Remark 2.3. In Section 6 below we consider the beta polymer model, which may also be viewed as a particle system, but the particles live in $(0,1]$. For concreteness, in the general discussion in this section we stick to particle systems in $\mathbb{Z}$.

\subsection{Coupling}

Let $m_{1}, m_{2}$ be two probability measures on the same measurable space $(\mathrm{E}, \mathcal{F})$. A coupling between $m_{1}$ and $m_{2}$ is, by definition, a measure $M=M\left(\mathrm{~d} z, \mathrm{~d} z^{\prime}\right)$ on $(\mathrm{E} \times \mathrm{E}, \mathcal{F} \otimes \mathcal{F})$ whose marginals are $m_{1}(\mathrm{~d} z)$ and $m_{2}\left(\mathrm{~d} z^{\prime}\right)$, respectively:

$$
\int_{z^{\prime} \in \mathrm{E}} M\left(\cdot, \mathrm{d} z^{\prime}\right)=m_{1}(\cdot), \quad \int_{z \in \mathrm{E}} M(\mathrm{~d} z, \cdot)=m_{2}(\cdot) .
$$

A coupling is not defined uniquely, but always exists (the product measure $M=m_{1} \otimes m_{2}$ is an example).

In the notation of the previous section, start from a parameter-symmetric particle system $\mathbf{x}^{\boldsymbol{\nu}}(t)$. Fix time $t$ and index $n \in \mathbb{Z}_{\geq 1}$, and consider two distributions $\mathbf{x}^{\boldsymbol{\nu}}(t)$ and $\mathbf{x}^{s_{n} \boldsymbol{\nu}}(t)$ on the same countable space $\operatorname{Conf}_{\text {fin }}(\mathbb{Z})$. We would like to find a coupling $M=M_{n}$ between the distributions of $\mathbf{x}^{\boldsymbol{\nu}}(t)$ and $\mathbf{x}^{s_{n} \boldsymbol{\nu}}(t)$ which satisfies an additional constraint corresponding to (2.1):

$$
M_{n}\left(x_{k}^{\nu}(t)=x_{k}^{s_{n} \nu}(t) \text { for all } k \in \mathbb{Z}_{\geq 1}, k \neq n\right)=1 .
$$

Such a coupling also might not be defined uniquely. An example of a coupling satisfying (2.2) can be obtained by adapting the basic product measure example. For any particle configuration $\mathbf{y}=\left(y_{1}, y_{2}, \ldots\right)$ denote $\mathbf{y}_{\hat{n}}:=\left\{y_{k}: k \neq n\right\}$. Define

$$
\begin{aligned}
& M_{n}^{\text {indep }}\left(\mathbf{x}^{\boldsymbol{\nu}}(t)=\mathbf{y}, \mathbf{x}^{s_{n} \boldsymbol{\nu}}(t)=\mathbf{z}\right) \\
& \quad:=\delta\left(\mathbf{y}_{\hat{n}}=\mathbf{z}_{\hat{n}}\right) P\left(\mathbf{x}_{\hat{n}}^{\boldsymbol{\nu}}(t)=\mathbf{y}_{\hat{n}}\right) P\left(x_{n}^{\boldsymbol{\nu}}(t)=y_{n} \mid \mathbf{x}_{\hat{n}}^{\boldsymbol{\nu}}(t)\right) P\left(x_{n}^{s_{n} \boldsymbol{\nu}}(t)=z_{n} \mid \mathbf{x}_{\hat{n}}^{s_{\hat{n}} \boldsymbol{\nu}}(t)\right) .
\end{aligned}
$$

Here $\delta(\cdot)$ is the Dirac delta, and the two quantities $P(\cdot \mid \cdot)$ are the conditional distributions

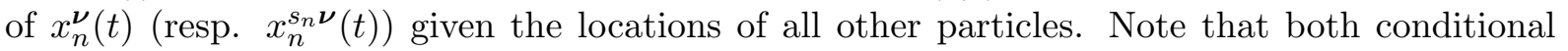
distributions $P(\cdot \mid \cdot)$ in $(2.3)$ are supported on the same interval

$$
I_{n}:=\left\{x_{n+1}^{\nu}(t)+1, x_{n+1}^{\nu}(t)+2, \ldots, x_{n-1}^{\nu}(t)-1\right\} \subset \mathbb{Z}
$$

(if $n=1$, then, by agreement, $x_{0} \equiv+\infty$ and the interval is infinite; for $n \geq 2$ the interval is finite). The next statement follows from the above definitions:

Lemma 2.4. The distribution $M_{n}^{\text {indep }}$ defined by (2.3) is a coupling between the distributions of $\mathbf{x}^{\boldsymbol{\nu}}(t)$ and $\mathbf{x}^{s_{n} \boldsymbol{\nu}}(t)$, and satisfies (2.2). 


\subsection{Swap operators}

With a coupling one can typically associate two conditional distributions. In our situation, a coupling $M_{n}$ satisfying (2.2) leads to two distributions on $I_{n}(2.4)$ which we denote by

$$
p_{n}=\operatorname{Law}\left(x_{n}^{s_{n} \boldsymbol{\nu}}(t) \mid \mathbf{x}^{\boldsymbol{\nu}}(t)\right) \quad \text { and } \quad p_{n}^{\prime}=\operatorname{Law}\left(x_{n}^{\nu}(t) \mid \mathbf{x}^{s_{n} \boldsymbol{\nu}}(t)\right) .
$$

Indeed, under, say, $p_{n}$ it suffices to specify only the conditional distribution of $x_{n}^{s_{n} \nu}(t)$, as all the other locations in $x^{s_{n} \nu}(t)$ stay the same. Thus, a coupling $M_{n}$ satisfying (2.2) is determined by either $p_{n}$ or $p_{n}^{\prime}$.

In the particular example $M_{n}^{\text {indep }}(2.3)$, the distribution $p_{n}$ simply corresponds to forgetting the previous location of $x_{n}^{\nu}(t)$, and selecting independently the new particle $x_{n}^{s_{n} \nu}(t) \in I_{n}$ (according to the distribution with the parameters $s_{n} \boldsymbol{\nu}$ ) given the remaining configuration $x_{\hat{n}}^{s_{n} \nu}(t)=x_{\hat{n}}^{\nu}(t)$. This distribution $p_{n}$ corresponding to $M_{n}^{\text {indep }}$ can be quite complicated as it may depend on the whole remaining configuration $\mathbf{x}_{\hat{n}}^{\nu}(t)$. This dependence may also nontrivially incorporate the time parameter $t$.

In this paper we describe specific integrable parameter-symmetric particle systems for which there exist much simpler conditional probabilities $p_{n}$ or $p_{n}^{\prime}$. Let us give a definition clarifying what we mean here by "simpler":

Definition 2.5. The conditional probability $p_{n}$ is said to be local if $p_{n}=\operatorname{Law}\left(x_{n}^{s_{n} \boldsymbol{\nu}}(t) \mid \mathbf{x}^{\boldsymbol{\nu}}(t)\right)$ depends only on $n, \nu$, and three particle locations $x_{n+1}^{\nu}(t), x_{n}^{\nu}(t), x_{n-1}^{\nu}(t)$. The definition for $p_{n}^{\prime}$ is analogous.

We will interpret the local conditional probability $p_{n}$ as a Markov operator. When applied, $p_{n}$ leads to a random move $x_{n}^{\nu}(t) \rightarrow x_{n}^{s_{n} \nu}(t)$ given $x_{n+1}^{\nu}(t), x_{n-1}^{\nu}(t)$. In distribution the application of $p_{n}$ is equivalent to the swapping of the parameters $\nu_{n} \leftrightarrow \nu_{n+1}$. Due to this interpretation, we will call $p_{n}$ the (Markov) swap operator.

In the examples we consider, swap operators will also be independent of $t$.

Remark 2.6. Typically, only one of the probabilities $p_{n}$ and $p_{n}^{\prime}$ can be local (and thus correspond to a swap operator). Indeed, assuming that $p_{n}$ is local, we can write

$$
\begin{aligned}
& p_{n}^{\prime}\left(x_{n}^{\boldsymbol{\nu}}(t)=y_{n} \mid \mathbf{x}^{s_{n} \boldsymbol{\nu}}(t)=\mathbf{z}\right) \\
& \quad=p_{n}\left(x_{n}^{s_{n} \boldsymbol{\nu}}(t)=z_{n} \mid x_{n+1}^{\boldsymbol{\nu}}(t)=y_{n+1}, x_{n}^{\boldsymbol{\nu}}(t)=y_{n}, x_{n-1}^{\nu}(t)=y_{n-1}\right) \frac{P\left(\mathbf{x}^{\boldsymbol{\nu}}(t)=\mathbf{y}\right)}{P\left(\mathbf{x}^{s_{n} \boldsymbol{\nu}}(t)=\mathbf{z}\right)}
\end{aligned}
$$

where $\mathbf{y}_{\hat{n}}=\mathbf{z}_{\hat{n}}$, and we also assume that the probability in the denominator is nonzero. If one wants $p_{n}^{\prime}$ to be local, too, it is necessary that the ratio of the probabilities $\frac{P\left(\mathbf{x}^{\nu}(t)=\mathbf{y}\right)}{P\left(\mathbf{x}^{s_{n} \nu}(t)=\mathbf{z}\right)}$ (in which $\mathbf{y}, \mathbf{z}$ differ only by the location of the $n$-th particle) depends only on the four particle locations $x_{n+1}^{\nu}(t), x_{n}^{\nu}(t), x_{n}^{s_{n} \boldsymbol{\nu}}(t), x_{n-1}^{\nu}(t)$. This (quite strong) condition on the ratio of the probabilities does not hold for the particle systems considered in the present paper. (In particular, using the explicit Rakos-Schütz formula [44] expressing transition probabilities in TASEP with particle-dependent speeds as determinants one can check that the condition fails for the usual TASEP.)

\section{Swap operators for $q$-Hahn TASEP}

In this section we examine the parameter symmetry and swap operators for the $q$-Hahn TASEP [42]. A multiparameter version of the process preserving its integrability is due to [14]. 


\subsection{The $q$-deformed beta-binomial distribution}

We first recall the definition and properties of the $q$-deformed beta-binomial distribution $\varphi_{q, \mu, \nu}$ from $[20,42]$. We use the standard notation for the $q$-Pochhammer symbol $(x ; q)_{k}=(1-x) \times$ $(1-q x) \cdots\left(1-q^{k-1} x\right), k \in \mathbb{Z}_{\geq 1}$ (by agreement, $(x ; q)_{0}=1$ ).

Everywhere throughout the paper we assume that the main parameter $q$ is between 0 and 1 .

Definition 3.1. For $m \in \mathbb{Z}_{\geq 0}$, consider the following distribution on $\{0,1, \ldots, m\}$ :

$$
\varphi_{q, \mu, \nu}(j \mid m)=\mu^{j} \frac{(\nu / \mu ; q)_{j}(\mu ; q)_{m-j}}{(\nu ; q)_{m}} \frac{(q ; q)_{m}}{(q ; q)_{j}(q ; q)_{m-j}}, \quad 0 \leq j \leq m .
$$

When $m=+\infty$, extend the definition as

$$
\varphi_{q, \mu, \nu}(j \mid \infty)=\mu^{j} \frac{(\nu / \mu ; q)_{j}}{(q ; q)_{j}} \frac{(\mu ; q)_{\infty}}{(\nu ; q)_{\infty}}, \quad j \in \mathbb{Z}_{\geq 0} .
$$

The distribution depends on $q$ and two other parameters $\mu, \nu$.

When $0 \leq \mu \leq 1$ and $\nu \leq \mu$, the weights $\varphi_{q, \mu, \nu}(j \mid m)$ are nonnegative. ${ }^{4}$ They also sum to one:

$$
\sum_{j=0}^{m} \varphi_{q, \mu, \nu}(j \mid m)=1, \quad m \in\{0,1, \ldots\} \cup\{+\infty\} .
$$

We will need two other properties of the weights given in the next two lemmas.

Lemma 3.2 ([2, 20]). The weights satisfy a symmetry property: for all $m, y \in \mathbb{Z}_{\geq 0}$ we have

$$
\sum_{j=0}^{m} q^{j y} \varphi_{q, \mu, \nu}(j \mid m)=\sum_{k=0}^{y} q^{k m} \varphi_{q, \mu, \nu}(k \mid y) .
$$

Similarly, for all $y \in \mathbb{Z}_{\geq 0}$, we have

$$
\sum_{j=0}^{\infty} q^{j y} \varphi_{q, \mu, \nu}(j \mid \infty)=\varphi_{q, \mu, \nu}(0 \mid y) .
$$

Define the following difference operator:

$$
\left(\nabla_{\mu, \nu} f\right)(n):=\frac{\mu-\nu}{1-\nu} f(n-1)+\frac{1-\mu}{1-\nu} f(n) .
$$

The next statement is a key property of the $q$-deformed beta binomial distribution which allows to simplify the action of certain operators defined through $\varphi_{q, \mu, \nu}$ on functions satisfying special boundary conditions. This is a manifestation of the connection of $\varphi_{q, \mu, \nu}$ to the coordinate Bethe ansatz, as developed in [42].

Lemma 3.3. Fix parameters $\nu_{i} \in(0,1), i \in \mathbb{Z}$. Let a function $f\left(n_{1}, \ldots, n_{m}\right)$ from $\mathbb{Z}^{m}$ to $\mathbb{C}$ satisfy the following two-body boundary conditions

$$
\begin{gathered}
\frac{\nu_{n_{i}}(1-q)}{1-q \nu_{n_{i}}} f\left(n_{1}, \ldots, n_{i}-1, n_{i+1}-1, \ldots, n_{m}\right)+\frac{q-\nu_{n_{i}}}{1-q \nu_{n_{i}}} f\left(n_{1}, \ldots, n_{i}, n_{i+1}-1, \ldots, n_{m}\right) \\
\quad+\frac{1-q}{1-q \nu_{n_{i}}} f\left(n_{1}, \ldots, n_{i}, n_{i+1}, \ldots, n_{m}\right)-f\left(n_{1}, \ldots, n_{i}-1, n_{i+1}, \ldots, n_{m}\right)=0
\end{gathered}
$$

\footnotetext{
${ }^{4}$ These conditions do not exhaust the full range of $(q, \mu, \nu)$ for which the weights are nonnegative. See, e.g., [14, Section 6.6.1] for additional families of parameters leading to nonnegative weights.
} 
for all $\vec{n} \in \mathbb{Z}^{m}$ such that for some $i \in\{1, \ldots, m\}, n_{i}=n_{i+1}$. (In (3.2), only the $i$-th and the $(i+1)$-st components of $\vec{n}$ are changed.) Then we have

$$
\prod_{i=1}^{m}\left[\nabla_{\mu, \nu_{n}}\right]_{i} f(\underbrace{n, n, \ldots, n}_{m})=\sum_{j=0}^{m} \varphi_{q, \mu, \nu_{n}}(j \mid m) f(\underbrace{n, \ldots, n}_{m-j}, \underbrace{n-1, \ldots, n-1}_{j}) .
$$

Here $\left[\nabla_{\mu, \nu_{n}}\right]_{i}$ is the operator (3.1) applied in the $i$-th variable.

Proof. This is based on the quantum (noncommutative) binomial [42, Theorem 1], and is a straightforward generalization of the equivalence of the free and true evolution equations [20, Proposition 1.8]. The only difference here is that $\nu_{i}$ 's are allowed to vary. However, as the application of the quantum binomial result depends only on the parameter $\nu_{n}$ associated to the particular $n$ in (3.3), we see that the claim readily holds.

\subsection{Multiparameter $q$-Hahn TASEP}

Here we recall the particle-inhomogeneous version of the $q$-Hahn TASEP from [14, Section 6.6]. Let

$$
\nu_{i} \in(0,1), \quad i \in \mathbb{Z}_{\geq 1}, \quad \gamma \in\left[1, \sup _{i} \nu_{i}^{-1}\right]
$$

be parameters. To make the system nontrivial, the $\nu_{i}$ 's should be uniformly bounded away from 1.

The $q$-Hahn TASEP starts from step and evolves in $\operatorname{Conf}_{\text {fin }}(\mathbb{Z})$ in discrete time $t \in \mathbb{Z}_{\geq 0}$. At each time moment, each particle $x_{i}$ independently jumps to the right by $j$ with probability

$$
\varphi_{q, \gamma \nu_{i}, \nu_{i}}\left(j \mid x_{i-1}-x_{i}-1\right), \quad j \in\left\{0,1, \ldots, x_{i-1}-x_{i}-1\right\},
$$

where $x_{0}=+\infty$, by agreement. See Fig. 1 for an illustration. For the step initial configuration the $q$-moments of the $q$-Hahn TASEP were obtained in [14, Corollary 10.4] (in the homogeneous case $\nu_{i} \equiv \nu$ a proof using duality and coordinate Bethe ansatz is due to [20]). The $q$-moments are given in the next proposition.

Proposition 3.4. For any $\ell \in \mathbb{Z}_{\geq 1}$ and any $n_{1} \geq n_{2} \geq \cdots \geq n_{\ell} \geq 1$ with the assumption that

$$
\min _{1 \leq i \leq n_{1}} \nu_{i}>q \max _{1 \leq i \leq n_{1}} \nu_{i}
$$

we have for the q-moments of the q-Hahn TASEP started from step:

$$
\begin{aligned}
\mathbb{E}_{\text {step }}^{\mathrm{qH}(\boldsymbol{\nu})} \prod_{j=1}^{\ell} q^{x_{n_{j}}(t)+n_{j}}= & (-1)^{\ell} q^{\frac{\ell(\ell-1)}{2}} \oint \frac{\mathrm{d} z_{1}}{2 \pi \mathbf{i}} \cdots \oint \frac{\mathrm{d} z_{\ell}}{2 \pi \mathbf{i}} \prod_{1 \leq A<B \leq \ell} \frac{z_{A}-z_{B}}{z_{A}-q z_{B}} \\
& \times \prod_{i=1}^{\ell}\left(\left(\frac{1-\gamma z_{i}}{1-z_{i}}\right)^{t} \frac{1}{z_{i}\left(1-z_{i}\right)} \prod_{j=1}^{n_{i}} \frac{1-z_{i}}{1-z_{i} / \nu_{j}}\right) .
\end{aligned}
$$

Here the integration contours are positively oriented simple closed curves which are q-nested around $\left\{\nu_{j}\right\}_{j=1, \ldots, n_{1}}$ (that is, each contour encircles the $\nu_{j}$ 's, and, moreover, the $z_{A}$ contour encircles each $q z_{B}$ contour, $B>A$ ) and leave 0 and 1 outside. See Fig. 3 for an illustration.

Remark 3.5. Together with particle-dependent inhomogeneity governed by the parameters $\nu_{i}$, one can make the parameter $\gamma$ time-dependent. That is, at each time step $t-1 \rightarrow t$, the jumping distribution (3.4) can be replaced by $\varphi_{q, \gamma_{t} \nu_{i}, \nu_{i}}\left(j \mid x_{i-1}-x_{i}-1\right)$. The moment formula (3.6) continues to hold when modified by replacing the term $\left(\frac{1-\gamma z_{i}}{1-z_{i}}\right)^{t}$ with $\prod_{l=1}^{t} \frac{1-\gamma_{l} z_{i}}{1-z_{i}}$. The main result of this section (Theorem 3.8 below) also holds in this generality, but for simplicity we will continue to assume that $\gamma$ does not depend on $t$. 


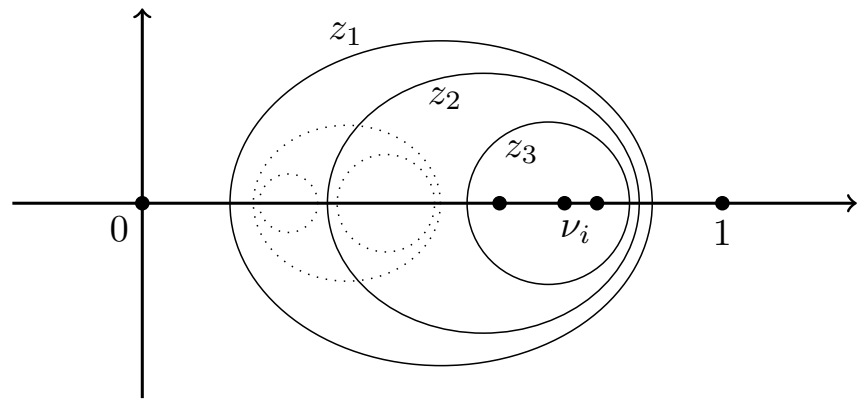

Figure 3. Possible integration contours in (3.6) for $\ell=3$. The contours for $q z_{3}, q^{2} z_{3}$, and $q z_{2}$ are shown dotted.

Since $0<q<1$ and we start from step, the random variables $\prod_{j=1}^{\ell} q^{x_{n_{j}}(t)+n_{j}}$ are all between 0 and 1. Because the moment problem for bounded random variables admits a unique solution, the $q$-moments (3.6) uniquely determine the joint distribution of all the $q$-Hahn TASEP particles $\left\{x_{i}(t)\right\}_{i \in \mathbb{Z}_{\geq 1}}$ at each fixed time moment. This implies the following statement:

Proposition 3.6. The multiparameter $q$-Hahn TASEP started from the step initial configuration is a parameter-symmetric particle system in the sense of Definition 2.1. Moreover, the distribution of each $x_{n}(t)$ depends on the parameters $\nu_{1}, \ldots, \nu_{n}$ in a symmetric way.

Denote the right-hand of $(3.6)$ by $f\left(n_{1}, \ldots, n_{\ell}\right)$, where now $n_{i} \in \mathbb{Z}$ are not necessarily ordered. Notice that if $n_{\ell}=0$, the integrand has no poles inside the $z_{\ell}$ (i.e., the smallest) integration contour. Therefore, $f\left(n_{1}, \ldots, n_{\ell-1}, 0\right)=0$. The following lemma will be employed in the next section.

Lemma 3.7. The function $f\left(n_{1}, \ldots, n_{\ell}\right)$ on $\mathbb{Z}^{\ell}$ defined before the lemma satisfies the two-body boundary conditions (3.2).

Proof. This statement essentially appears in [20], see also [10]. Its proof is rather short so we reproduce it here. When $n_{i}=n_{i+1}$ (denote them both by $n$ ), the part of the integrand in (3.6) depending on $z_{i}, z_{i+1}$ contains

$$
\frac{z_{i}-z_{i+1}}{z_{i}-q z_{i+1}} \prod_{j=1}^{n} \frac{\left(1-z_{i}\right)\left(1-z_{i+1}\right)}{\left(1-z_{i} / \nu_{j}\right)\left(1-z_{i+1} / \nu_{j}\right)} .
$$

The left-hand side of the boundary conditions (3.2) for our function $f$ is an integral over the contours as in Fig. 3, where the integrand now contains

$$
\begin{aligned}
\frac{z_{i}-z_{i+1}}{z_{i}-q z_{i+1}} & \prod_{j=1}^{n-1} \frac{\left(1-z_{i}\right)\left(1-z_{i+1}\right)}{\left(1-z_{i} / \nu_{j}\right)\left(1-z_{i+1} / \nu_{j}\right)} \\
& \times\left(\frac{\nu_{n}(1-q)}{1-q \nu_{n}}+\frac{q-\nu_{n}}{1-q \nu_{n}} \frac{1-z_{i}}{1-z_{i} / \nu_{n}}+\frac{1-q}{1-q \nu_{n}} \frac{1-z_{i}}{1-z_{i} / \nu_{n}} \frac{1-z_{i+1}}{1-z_{i+1} / \nu_{n}}-\frac{1-z_{i+1}}{1-z_{i+1} / \nu_{n}}\right) \\
& =\frac{\nu_{n}\left(1-\nu_{n}\right)^{2}}{1-q \nu_{n}} \frac{z_{i}-z_{i+1}}{\left(z_{i}-\nu_{n}\right)\left(z_{i+1}-\nu_{n}\right)} \prod_{j=1}^{n-1} \frac{\left(1-z_{i}\right)\left(1-z_{i+1}\right)}{\left(1-z_{i} / \nu_{j}\right)\left(1-z_{i+1} / \nu_{j}\right)},
\end{aligned}
$$

where the important observation is that the denominator $z_{i}-q z_{i+1}$ has canceled out. Now the contour for $z_{i}$ can be deformed (without picking any residues) to coincide with the contour for $z_{i+1}$. However, thanks to the factor $z_{i}-z_{i+1}$, the integrand is antisymmetric in $z_{i}, z_{i+1}$. Therefore, the whole integral vanishes, as desired. 


\subsection{Markov swap operators for $q$-Hahn TASEP}

Here we prove that the $q$-Hahn TASEP admits a local conditional distribution corresponding to the permutation $s_{n}=(n, n+1)$ when the parameters satisfy $\nu_{n+1}<\nu_{n}$ before their swap. This leads to the Markov swap operator which we define now. Fix $n \in \mathbb{Z}_{>1}$, and let

$$
p_{n}^{\mathrm{qH}}\left(x_{n}^{\prime} \mid x_{n+1}, x_{n}, x_{n-1}\right):=\varphi_{q, \frac{\nu_{n+1}}{\nu_{n}}, \nu_{n+1}}\left(x_{n}^{\prime}-x_{n+1}-1 \mid x_{n}-x_{n+1}-1\right) .
$$

Observe that this probability does not depend on $x_{n-1}$. The condition $\nu_{n+1}<\nu_{n}$ ensures that the swap operator $p_{n}^{\mathrm{qH}}(3.7)$ has nonnegative probability weights.

Theorem 3.8 (Theorem 1.1 in Introduction). Let $\mathbf{x}^{\boldsymbol{\nu}}(t)$ be the q-Hahn TASEP with parameters $\boldsymbol{\nu}=\left\{\nu_{i}\right\}_{i \in \mathbb{Z}_{>1}}$, started from step. Fix $n \in \mathbb{Z}_{\geq 1}$ and assume that $\nu_{n+1}<\nu_{n}$. Replace $x_{n}(t)$ by a random $x_{n}^{\prime}(t)$ coming from the Markov swap operator $p_{n}^{\mathrm{qH}}$ (3.7). Then the new configuration is distributed as the $q$-Hahn TASEP $\mathbf{x}^{s_{n} \nu}(t)$ with swapped parameters.

Proof. We will prove this theorem by applying $p_{n}^{\mathrm{qH}}$ in the $q$-moment formula. Since the $q$-moments uniquely determine the distribution, this computation will imply the claim.

Fix integers $\ell, \ell^{\prime}, a, b \geq 0$ and define

$$
\vec{n}=\left(n_{1}, \ldots, n_{k}\right):=(m_{1}, \ldots, m_{\ell}, \underbrace{n+1, \ldots, n+1}_{a}, \underbrace{n, \ldots, n}_{b}, m_{1}^{\prime}, \ldots, m_{\ell^{\prime}}^{\prime}),
$$

where $m_{1} \geq \cdots \geq m_{\ell}>n+1, n>m_{1}^{\prime} \geq \cdots \geq m_{\ell^{\prime}}^{\prime} \geq 1$, and $k=\ell+a+b+\ell^{\prime}$. Assume that the parameters $\nu_{i}$ satisfy the contour existence condition (3.5). (In the end of the proof we will drop this assumption.) It suffices to show that

$$
\mathbb{E}_{\text {step }}^{\mathrm{qH}(\boldsymbol{\nu})}\left(\sum_{x_{n}^{\prime}=x_{n+1}(t)+1}^{x_{n}(t)} p_{n}^{\mathrm{qH}}\left(x_{n}^{\prime} \mid x_{n+1}(t), x_{n}(t), x_{n-1}(t)\right) q^{b\left(x_{n}^{\prime}+n\right)} \prod_{\substack{j=1 \\ n_{j} \neq n}}^{k} q^{x_{n_{j}}(t)+n_{j}}\right)
$$

(where the expectation $\mathbb{E}_{\text {step }}^{\mathrm{qH}(\boldsymbol{\nu})}$ is taken with the parameters before the swap), is equal to the expectation

$$
\mathbb{E}_{\text {step }}^{\mathrm{qH}\left(s_{n} \boldsymbol{\nu}\right)} \prod_{j=1}^{k} q^{x_{n_{j}}(t)+n_{j}}
$$

with the swapped parameters. Indeed, the sum over $x_{n}^{\prime}$ in (3.9) corresponds to the action of the swap operator $p_{n}^{\mathrm{qH}}$ on $\prod_{j=1}^{k} q^{x_{n_{j}}(t)+n_{j}}$ viewed as a function of $\left\{x_{i}(t)\right\}$. We thus need to show that the expectation of the result with respect to the original parameters leads to the formula with the swapped parameters.

We now start from (3.9), and in the rest of the proof omit the dependence on $t$ for shorter notation. First, we use the symmetry property (Lemma 3.2) to write for the part of the sum in (3.9) involving $x_{n}, x_{n+1}$ :

$$
\begin{aligned}
& \sum_{x_{n}^{\prime}=x_{n+1}+1}^{x_{n}} \varphi_{q, \frac{\nu_{n+1}}{\nu_{n}}, \nu_{n+1}}\left(x_{n}^{\prime}-x_{n+1}-1 \mid x_{n}-x_{n+1}-1\right) q^{a\left(x_{n+1}+n+1\right)+b\left(x_{n}^{\prime}+n\right)} \\
& \quad=\sum_{x_{n}^{\prime}=x_{n+1}+1}^{x_{n}} q^{b\left(x_{n}^{\prime}-x_{n+1}-1\right)} \varphi_{q, \frac{\nu_{n+1}}{\nu_{n}}, \nu_{n+1}}\left(x_{n}^{\prime}-x_{n+1}-1 \mid x_{n}-x_{n+1}-1\right) q^{(a+b)\left(x_{n+1}+n+1\right)} \\
& =\sum_{r=0}^{b} q^{r\left(x_{n}-x_{n+1}-1\right)} \varphi_{q, \frac{\nu_{n+1}}{\nu_{n}}, \nu_{n+1}}(r \mid b) q^{(a+b)\left(x_{n+1}+n+1\right)} \\
& =\sum_{r=0}^{b} \varphi_{q, \frac{\nu_{n+1}}{\nu_{n}}, \nu_{n+1}}(r \mid b) q^{(a+b-r)\left(x_{n+1}+n+1\right)+r\left(x_{n}+n\right)} .
\end{aligned}
$$


We thus need to compute

$$
\sum_{r=0}^{b} \varphi_{q, \frac{\nu_{n+1}}{\nu_{n}}, \nu_{n+1}}(r \mid b) \mathbb{E}_{\text {step }}^{\mathrm{qH}(\boldsymbol{\nu})}\left(\prod_{j=1}^{k} q^{x_{n_{j}(r)}+n_{j}(r)}\right),
$$

where the vector $\vec{n}(r)=\left(n_{1}(r), \ldots, n_{k}(r)\right)$ is as in (3.8), but with $(a, b)$ replaced by $(a+b-r, r)$. The expectation in (3.12) is given by the contour integral as in the right-hand side of (3.6). Recall the notation $f(\vec{n})$ for this integral, where now $\vec{n} \in \mathbb{Z}^{k}$, and the components of $\vec{n}$ are not necessarily ordered. By Lemma 3.7, this function $f$ satisfies the two-body boundary conditions. Thus, (3.12) can be rewritten by Lemma 3.3 as (recall notation (3.1) for the operator $\nabla_{\mu, \nu}$ ):

$$
\prod_{j=1}^{b}\left[\nabla_{\frac{\nu_{n+1}}{\nu_{n}}, \nu_{n+1}}\right]_{\ell+a+j} f(m_{1}, \ldots, m_{\ell}, \underbrace{n+1, \ldots, n+1}_{a+b}, m_{1}^{\prime}, \ldots, m_{\ell^{\prime}}^{\prime}) .
$$

Observe that now each of the difference operators $\left[\frac{\nu_{n+1}}{\nu_{n}}, \nu_{n+1}\right] \ell+a+j$ can be applied independently inside the integral. We thus have for every variable $w=z_{\ell+a+j}, j=1, \ldots, b$ :

$$
\begin{aligned}
{\left[\nabla_{\frac{\nu_{n+1}}{\nu_{n}}, \nu_{n+1}}\right]_{\ell+a+j} \prod_{i=1}^{n+1} \frac{1-w}{1-w / \nu_{i}} } & =\left(\frac{\nu_{n+1} / \nu_{n}-\nu_{n+1}}{1-\nu_{n+1}}+\frac{1-\nu_{n+1} / \nu_{n}}{1-\nu_{n+1}} \frac{1-w}{1-w / \nu_{n+1}}\right) \prod_{i=1}^{n} \frac{1-w}{1-w / \nu_{i}} \\
& =\frac{1-w / \nu_{n}}{1-w / \nu_{n+1}} \prod_{i=1}^{n} \frac{1-w}{1-w / \nu_{i}}=\prod_{\substack{i=1 \\
i \neq n}}^{n+1} \frac{1-w}{1-w / \nu_{i}} .
\end{aligned}
$$

We see that the resulting integral coming from (3.9) contains, for each variable $z_{\ell+a+j}$ corresponding to $n_{\ell+a+j}=n$ in (3.8), the product over the parameters $\left(\nu_{1}, \ldots, \nu_{n-1}, \nu_{n+1}\right)=$ $s_{n}\left(\nu_{1}, \ldots, \nu_{n}\right)$. Therefore, this integral is equal to the expectation (3.10) with the swapped parameters $s_{n} \boldsymbol{\nu}$, as desired.

It remains to show that we can drop the contour existence assumption (3.5). The preceding argument implies that under (3.5) (with fixed $x_{n+1}, x_{n}^{\prime}, x_{n-1}$ ),

$$
\begin{aligned}
& \sum_{x_{n}=x_{n}^{\prime}}^{x_{n-1}-1} P_{\nu}\left(\ldots, x_{n+1}, x_{n}, x_{n-1}, \ldots\right) \varphi_{q, \frac{\nu_{n+1}}{\nu_{n}}, \nu_{n+1}}\left(x_{n}^{\prime}-x_{n+1}-1 \mid x_{n}-x_{n+1}-1\right) \\
& \quad=P_{s_{n}}\left(\ldots, x_{n+1}, x_{n}^{\prime}, x_{n-1}, \ldots\right),
\end{aligned}
$$

where $P_{\nu}, P_{s_{n} \nu}$ denote the $q$-Hahn probability distributions with the corresponding parameters at some fixed time $t \in \mathbb{Z}_{\geq 0}$.

If $n \geq 2$, the sum in the left-hand side of (3.13) is finite, and each probability $P_{\boldsymbol{\nu}}, P_{s_{n} \boldsymbol{\nu}}$ is a rational function of $\nu_{2}, \nu_{3}, \ldots$ (note that since $\ldots, x_{n+1}, x_{n}^{\prime}, x_{n-1}, \ldots$, are fixed, only finitely many of the $\nu_{i}$ 's enter (3.13)). The dependence on $\nu_{1}$ is also rational after canceling out the common factor $\frac{\left(\gamma \nu_{1} ; q\right)_{\infty}^{t}}{\left(\nu_{1} ; q\right)_{\infty}^{t}}$ from both sides. Therefore, identity (3.13) between rational functions in $\nu_{i}$ can be analytically continued, and the assumption (3.5) can be dropped.

For $n=1$, the sum in the left-hand side of (3.13) becomes infinite. Remove the common factor $\frac{\left(\gamma \nu_{1} ; q\right)_{\infty}^{t}}{\left(\nu_{1} ; q\right)_{\infty}^{t}}$ from both sides again, then the coefficients by each power $\gamma^{m}, m \in \mathbb{Z}_{\geq 0}$, become rational functions in $\nu_{i}, i=1,2, \ldots$. Therefore, we can again analytically continue identity (3.13) and drop the assumption (3.5). This completes the proof.

Remark 3.9. When $\nu_{n}=\nu_{n+1}$, we have from (3.7) that $p_{n}^{\mathrm{qH}}\left(x_{n}^{\prime} \mid x_{n+1}, x_{n}, x_{n-1}\right)=\mathbf{1}_{x_{n}^{\prime}=x_{n}}$ (where $1 \ldots$ stands for the indicator). Therefore, the swap operator reduces to the identity map, which is appropriate since for $\nu_{n}=\nu_{n+1}$ there is nothing to swap. 
If $\nu_{n}<\nu_{n+1}$, formula (3.7) for $p_{n}^{\mathrm{qH}}$ also makes sense, but some of these probability weights become negative. One can check that all algebraic manipulations in the proof of Theorem 3.8 are still valid for $\nu_{n}<\nu_{n+1}$, but now they do not correspond to actual stochastic objects. This is the reason for the restriction $\nu_{n}>\nu_{n+1}$ in Theorem 3.8.

\subsection{Duality for the $q$-Hahn swap operator}

Here let us recall the Markov duality relation for the $q$-Hahn TASEP from [20]. We will heavily use duality in Section 5 below.

Fix $\ell \geq 1$ and let

$$
\mathbb{W}^{\ell}:=\left\{\vec{n}=\left(n_{1} \geq \cdots \geq n_{\ell} \geq 0\right), n_{i} \in \mathbb{Z}\right\} .
$$

We interpret elements of $\mathbb{W}^{\ell}$ as $\ell$-particle configurations in $\mathbb{Z}_{\geq 0}$, where multiple particles per site are allowed. Namely, for each $i=1, \ldots, \ell$, we put one particle at the location $n_{i} \in \mathbb{Z}_{\geq 0}$. See Fig. 4 for an illustration.

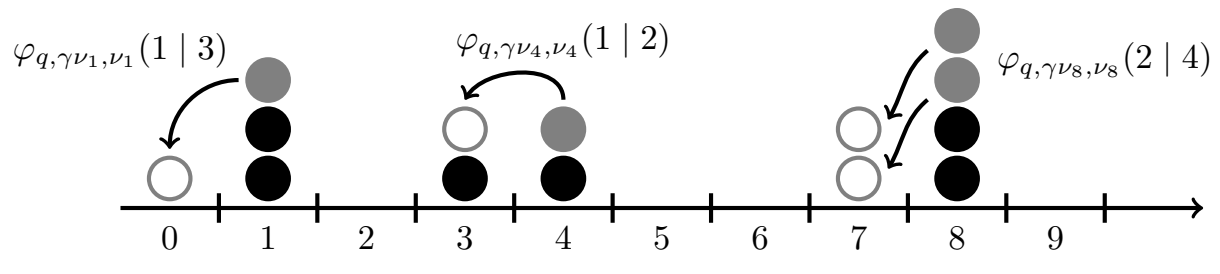

Figure 4. Configuration of particles $\vec{n}=(8,8,8,8,4,4,3,1,1,1) \in \mathbb{W}^{10}$, and a possible one-step transition in the $q$-Hahn Boson process. The particles of $\vec{n}$ which jump are solid gray, and their new locations are not filled.

Define the duality functional on the product space $\operatorname{Conf}_{\text {fin }}(\mathbb{Z}) \times \mathbb{W}^{\ell}$ as follows:

$$
H(\mathbf{x}, \vec{n}):= \begin{cases}\prod_{i=1}^{\ell} q^{x_{n_{i}}+n_{i}}, & n_{\ell} \geq 1, \\ 0, & n_{\ell}=0 .\end{cases}
$$

Let $\mathcal{T}^{\mathrm{qH}(\boldsymbol{\nu})}(\mathbf{x}, \mathbf{y}), \mathbf{x}, \mathbf{y} \in \operatorname{Conf}_{\text {fin }}(\mathbb{Z})$, denote the one-step Markov transition operator of the $q$-Hahn TASEP with parameters $\boldsymbol{\nu}=\left\{\nu_{i}\right\}$ and $\gamma$. We do not include the latter in the notation and assume that it is fixed throughout this section.

Let $\breve{\mathcal{T}}^{\mathrm{qH}(\boldsymbol{\nu})}(\vec{n}, \vec{m}), \vec{n}, \vec{m} \in \mathbb{W}^{\ell}$, be the one-step transition operator of a discrete time Markov chain on $\mathbb{W}^{\ell}$ which at each time step evolves as follows. Independently at every site $k \in \mathbb{Z}_{>1}$ containing, say, $y_{k}$ particles, randomly select $j$ particles with probability $\varphi_{q, \gamma \nu_{k}, \nu_{k}}\left(j \mid y_{k}\right)$, and move them to the site $k-1$. This Markov chain is called the (stochastic) q-Hahn Boson process. See Fig. 4 for an illustration.

Proposition 3.10 ([20]). With the above definitions, we have

$$
\mathcal{T}^{\mathrm{qH}(\boldsymbol{\nu})} H(\mathbf{x}, \vec{n})=\breve{\mathcal{T}}^{\mathrm{qH}(\boldsymbol{\nu})} H(\mathbf{x}, \vec{n}), \quad \mathbf{x} \in \operatorname{Conf}_{\text {fin }}(\mathbb{Z}), \quad \vec{n} \in \mathbb{W}^{\ell} .
$$

Here the operators $\mathcal{T}^{\mathrm{qH}(\boldsymbol{\nu})}, \breve{\mathcal{T}}^{\mathrm{qH}(\boldsymbol{\nu})}$ act in the $\mathbf{x}$ and the $\vec{n}$ variables, respectively. Equivalently in terms of expectations, we have for all $\mathbf{x}^{0} \in \operatorname{Conf}_{\text {fin }}(\mathbb{Z}), \vec{n}^{0} \in \mathbb{W}^{\ell}$, and all times $t \in \mathbb{Z}_{\geq 0}$ :

$$
\mathbb{E}_{\mathbf{x}(0)=\mathbf{x}^{0}}^{\mathrm{qH}(\boldsymbol{\nu})} H\left(\mathbf{x}(t), \vec{n}^{0}\right)=\mathbb{E}_{\vec{n}(0)=\vec{n}^{0}}^{\mathrm{qHBos}(\boldsymbol{\nu})} H\left(\mathbf{x}^{0}, \vec{n}(t)\right) .
$$

Here in the left-hand side the expectation is taken with respect to the q-Hahn TASEP's evolution starting from $\mathbf{x}^{0}$, and in the right-hand side the expectation is with respect to the q-Hahn Boson process started from $\vec{n}^{0}$. 
Consider now the Markov swap operator $p_{k}^{\mathrm{qH}}(3.7)$ on $\operatorname{Conf}_{\text {fin }}(\mathbb{Z})$, where $k \in \mathbb{Z}_{\geq 1}$ is fixed, and $\nu_{k}>\nu_{k+1}$. It turns out that this operator admits a dual Markov operator on the space $\mathbb{W}^{\ell}$, by means of the same duality functional $H$ (3.15). Namely, define $\breve{p}_{k}^{\mathrm{qH}}$ as the Markov operator which acts only on the $k$-th location in the $q$-Boson configuration. If the $k$-th location contains $y_{k}$ particles, then $\breve{p}_{k}^{\mathrm{qH}}$ randomly sends $y_{k}-j$ particles from location $k$ to location $k+1$, with probability $\varphi_{q, \frac{\nu_{k+1}}{\nu_{k}}, \nu_{k+1}}\left(j \mid y_{k}\right)$. See Fig. 5 for an illustration.

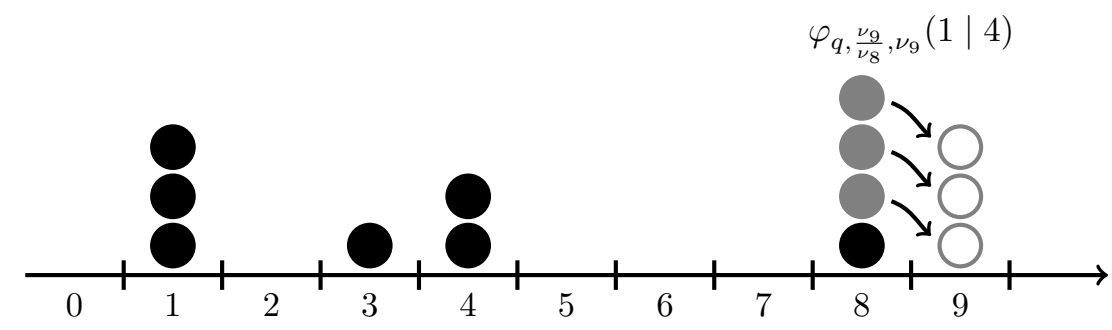

Figure 5. A possible transition under $\breve{p}_{8}^{\mathrm{qH}}$.

Proposition 3.11. If $\nu_{k}>\nu_{k+1}$, then we have

$p_{k}^{\mathrm{qH}} H(\mathbf{x}, \vec{n})=\breve{p}_{k}^{\mathrm{qH}} H(\mathbf{x}, \vec{n})$,

where the operator in the left-hand side acts on $\mathbf{x}$, and in the right-hand side - on $\vec{n}$.

Proof. This duality relation immediately follows from computation (3.11) performed (with the help of Lemma 3.2) in the proof of Theorem 3.8.

\section{Continuous time limit of repeated swaps}

Here we consider two continuous time limits, one of the original $q$-Hahn TASEP, and another one of the transition probabilities $p_{n}^{\mathrm{qH}}$ leading to the new backward $q$-Hahn process. These two continuous time processes act on the two-parameter family $\left\{\mathcal{N}_{q, \nu ; \mathrm{t}}^{\mathrm{qH}}\right\}_{\mathrm{t} \in \mathbb{R}_{\geq 0}, 0 \leq \nu<1}$ of distributions of the continuous time $q$-Hahn TASEP (started from step) by continuously changing the parameters.

\subsection{Two expansions of the distribution $\varphi_{q, \mu, \nu}$}

Let us write down two Taylor expansions of the $q$-deformed beta-binomial distribution from Section 3.1. Their proofs are straightforward.

Lemma 4.1. For $\nu \in[0,1)$ and $m \in\{1,2, \ldots,\} \cup\{+\infty\}$ we have as $\varepsilon \searrow 0$ :

$$
\varphi_{q, \nu+\varepsilon, \nu}(j \mid m)= \begin{cases}1+O(\varepsilon), & j=0, \\ \frac{\nu^{j-1}}{1-q^{j}} \frac{(q ; q)_{m}(\nu ; q)_{m-j}}{(q ; q)_{m-j}(\nu ; q)_{m}} \varepsilon+O\left(\varepsilon^{2}\right), & 1 \leq j \leq m .\end{cases}
$$

Lemma 4.2. For $\nu \in[0,1)$ and $m \in\{0,1,2, \ldots\}$ we have as $\varepsilon \searrow 0$ :

$$
\varphi_{q, 1-\varepsilon, \nu(1-\varepsilon)}(j \mid m)= \begin{cases}\frac{1}{1-q^{m-j}} \frac{(q ; q)_{m}(\nu ; q)_{j}}{(q ; q)_{j}(\nu ; q)_{m}} \varepsilon+O\left(\varepsilon^{2}\right), & 0 \leq j \leq m-1, \\ 1+O(\varepsilon), & j=m .\end{cases}
$$


Denote

$$
\psi_{q, \nu}(j \mid m):=\frac{\nu^{j-1}}{1-q^{j}} \frac{(q ; q)_{m}(\nu ; q)_{m-j}}{(q ; q)_{m-j}(\nu ; q)_{m}}, \quad \psi_{q, \nu}^{\bullet}\left(j^{\prime} \mid m\right):=\frac{1}{1-q^{m-j^{\prime}}} \frac{(q ; q)_{m}(\nu ; q)_{j^{\prime}}}{(q ; q)_{j^{\prime}}(\nu ; q)_{m}}
$$

where $1 \leq j \leq m$ and $0 \leq j^{\prime} \leq m-1$. Clearly, $\psi_{q, \nu}(j \mid m)=\nu^{j-1} \psi_{q, \nu}^{\bullet}(m-j \mid m)$ for all $1 \leq j \leq m$, but it is convenient to keep these notations separate.

\subsection{Continuous time $q$-Hahn TASEP}

Fix $q \in(0,1), \nu \in[0,1)$ and consider the Poisson-type limit of the $q$-Hahn TASEP with homogeneous parameters $\nu_{i} \equiv \nu$ as

$$
\gamma=1+\varepsilon / \nu, \quad t=\lfloor\mathrm{t} / \varepsilon\rfloor, \quad \varepsilon \searrow 0,
$$

where $t \in \mathbb{Z}_{\geq 0}$ is the discrete time before the limit, and $t \in \mathbb{R}_{\geq 0}$ is the scaled continuous time after the limit. The resulting process is the continuous time q-Hahn TASEP which evolves as follows. Starting from step, in continuous time $\mathrm{t} \in \mathbb{R}_{\geq 0}$, each particle $x_{n}(\mathrm{t}), n \in \mathbb{Z}_{\geq 1}$, independently jumps to the right by $j \in\left\{1,2, \ldots, x_{n-1}(\mathrm{t})-x_{n}(\mathrm{t})-1\right\}$ at rate

$$
\psi_{q, \nu}\left(j \mid x_{n-1}(\mathrm{t})-x_{n}(\mathrm{t})-1\right) .
$$

(In continuous time there is at most one jump at every instance of time.) Here $x_{0} \equiv+\infty$, by agreement. This continuous time process was considered in [3, 48].

Remark 4.3 (multiparameter continuous time $q$-Hahn TASEP). One can also consider a version of the continuous time $q$-Hahn TASEP in which each particle $x_{n}$ jumps at rate $\nu_{n} \psi_{q, \nu_{n}}(\cdot \mid \cdot)$. This multiparameter deformation preserves integrability. To get from the multiparameter process to the homogeneous one described above one has to set $\nu_{n} \equiv \nu$ and rescale the continuous time by the factor of $\nu$. For simpler notation, we will mostly consider the homogeneous continuous time $q$-Hahn TASEP. Its multiparameter generalization is needed only in the proof of Theorem 4.7 below.

The continuous time $q$-Hahn TASEP possesses the same $q$-moment formulas as (3.6), with $\nu_{i} \equiv \nu$, and the replacement

$$
\left(\frac{1-\gamma z_{i}}{1-z_{i}}\right)^{t} \rightarrow \exp \left\{-\frac{\mathrm{t}}{\nu} \frac{z_{i}}{1-z_{i}}\right\}, \quad i=1, \ldots, \ell
$$

inside the contour integral.

\subsection{Backward $q$-Hahn process}

Here we define a continuous time limit of a certain combination of the swap operators $p_{n}^{\mathrm{qH}}(3.7)$. Let us first explain the main idea. Assume that $\nu_{1}>\nu_{2}>\nu_{3}>\cdots$. By Theorem 3.8, the application of the Markov operators $p_{1}^{\mathrm{qH}}, p_{2}^{\mathrm{qH}}, \ldots$ (in this order) to a random configuration coming from the discrete time $q$-Hahn TASEP with parameters $\boldsymbol{\nu}$ is equivalent in distribution to the permutation $\boldsymbol{\nu} \mapsto \cdots s_{3} s_{2} s_{1} \boldsymbol{\nu}$ which exchanges $\nu_{1}$ with $\nu_{2}$, then $\nu_{1}$ with $\nu_{3}$, and so on (so that $\nu_{1}$ gets pushed all the way to infinity and essentially disappears). Because the particle configuration to which we apply the $p_{i}^{\mathrm{qH}}$, s is densely packed to the left, this application of the infinite product of the swap operators $p_{i}^{\mathrm{qH}}$ is well-defined and is a one-step Markov transition operator on $\operatorname{Conf}_{\text {fin }}(\mathbb{Z})$. Its continuous time limit as $\nu_{i} \rightarrow \nu$ for all $i$ will be our new backward $q$-Hahn TASEP. 
Let us now make this idea precise and take the particular parameters $\nu_{i}=\nu r^{i-1}, i \in \mathbb{Z}_{\geq 0}$, where $\nu \in[0,1)$ and $r \in(0,1)$. Denote by $p_{n}^{\mathrm{qH} ;(\alpha, \beta)}, 0<\beta<\alpha$, the Markov swap operator acting on the $n$-th particle as follows

$$
p_{n}^{\mathrm{qH} ;(\alpha ; \beta)}\left(x_{n}^{\prime} \mid x_{n+1}, x_{n}, x_{n-1}\right)=\varphi_{q, \frac{\beta}{\alpha}, \beta}\left(x_{n}^{\prime}-x_{n+1}-1 \mid x_{n}-x_{n+1}-1\right) .
$$

Then define the infinite product

$$
\mathcal{B}_{q, \nu}^{\mathrm{qH}(r)}:=p_{1}^{\mathrm{qH} ;\left(\nu_{2}, \nu_{1}\right)} p_{2}^{\mathrm{qH} ;\left(\nu_{3}, \nu_{1}\right)} p_{3}^{\mathrm{qH} ;\left(\nu_{4}, \nu_{1}\right)} \cdots=p_{1}^{\mathrm{qH} ;(\nu r, \nu)} p_{2}^{\mathrm{qH} ;\left(\nu r^{2}, \nu\right)} p_{3}^{\mathrm{qH} ;\left(\nu r^{3}, \nu\right)} \cdots
$$

(these are Markov operators so their product is written as if it's the action on measures: we first apply $p_{1}^{\mathrm{qH}}$, then $p_{2}^{\mathrm{qH}}$, and so on). In words, under the Markov operator $\mathcal{B}_{q, \nu}^{\mathrm{qH}(r)}$, each particle $x_{n}$ jumps to the left into a new location $x_{n}^{\prime} \in\left\{x_{n+1}+1, x_{n+1}+2, \ldots, x_{n}\right\}$ chosen randomly from the distribution

$$
\varphi_{q, r^{n}, \nu r^{n}}\left(x_{n}^{\prime}-x_{n+1}-1 \mid x_{n}-x_{n+1}-1\right) .
$$

The update is sequential for $n=1,2, \ldots$, so the new location $x_{n}^{\prime}$ of each $x_{n}$ depends only on the two old locations $x_{n+1}, x_{n}$.

Proposition 4.4. For $\nu_{i}=\nu r^{i-1}$ and any $m, k \in \mathbb{Z}_{\geq 0}$ we have

$$
\delta_{\text {step }}\left(\mathcal{T}^{\mathrm{qH}(\boldsymbol{\nu})}\right)^{m} \mathcal{B}_{q, \nu}^{\mathrm{qH}(r)} \mathcal{B}_{q, r \nu}^{\mathrm{qH}(r)} \mathcal{B}_{q, r^{2} \nu}^{\mathrm{qH}(r)} \cdots \mathcal{B}_{q, r^{k-1} \nu}^{\mathrm{qH}(r)}=\delta_{\text {step }}\left(\mathcal{T}^{\mathrm{qH}\left(r^{k} \boldsymbol{\nu}\right)}\right)^{m},
$$

where $\mathcal{T}^{\mathrm{qH}(\boldsymbol{\nu})}$ is the one-step Markov transition operator of the discrete time q-Hahn TASEP, $\delta_{\text {step }}$ is the delta measure at the step configuration, and $r^{k} \boldsymbol{\nu}=\left\{\nu r^{k+i-1}\right\}_{i \in \mathbb{Z}_{\geq 1}}$ is the parameter sequence shifted by $k$.

Proof. Follows from Theorem 3.8.

Note that $\mathcal{T}^{\mathrm{qH}\left(r^{k} \boldsymbol{\nu}\right)}$, the $q$-Hahn TASEP transition operator with the shifted parameter sequence $r^{k} \boldsymbol{\nu}$ from Proposition 4.4, is the same as $\left.\mathcal{T}^{\mathrm{qH}(\boldsymbol{\nu})}\right|_{\nu \mapsto r^{k} \nu}$, i.e., the original $q$-Hahn TASEP operator in which $\nu$ is replaced by $r^{k} \nu$.

Proposition 4.5. In the scaling regime

$$
r=1-\varepsilon, \quad k=\lfloor\tau / \varepsilon\rfloor, \quad k^{\prime}=\left\lfloor\tau^{\prime} / \varepsilon\right\rfloor, \quad \varepsilon \searrow 0,
$$

where $0 \leq \tau \leq \tau^{\prime}$ are scaled times, and the parameters $q, \nu$ are assumed fixed, we have

$$
\lim _{\varepsilon \searrow 0}\left(\mathcal{B}_{q, r^{k} \nu}^{\mathrm{qH}(r)} \mathcal{B}_{q, r^{k+1} \nu}^{\mathrm{qH}(r)} \mathcal{B}_{q, r^{k+2} \nu}^{\mathrm{qH}(r)} \cdots \mathcal{B}_{q, r^{k^{\prime}-2} \nu}^{\mathrm{qH}(r)} \mathcal{B}_{q, r^{k^{\prime}-1} \nu}^{\mathrm{qH}(r)}\right)=\mathcal{B}_{q, \nu}^{\mathrm{qH}}\left(\tau, \tau^{\prime}\right)
$$

as Markov operators acting on the space $\operatorname{Conf}_{\text {fin }}(\mathbb{Z})$. The convergence is in the sense of the operators' matrix elements (i.e., the strong operator topology). The operators $\mathcal{B}_{q, \nu}^{\mathrm{qH}}\left(\tau, \tau^{\prime}\right)$ form a continuous time, time-inhomogeneous Markov semigroup.

The fact that the resulting continuous time Markov chain is time-inhomogeneous will become clearer later in Section 4.4 when we consider its action on the $q$-Hahn TASEP distributions.

Proof of Proposition 4.5. Observe that the space of left-packed configurations $\operatorname{Conf}_{\text {fin }}(\mathbb{Z})$ is countable, and under the Markov operators in both sides of (4.3) the particles jump only to the left. Therefore, the desired limit as $\varepsilon \searrow 0$ reduces to the limit of finite-size Markov transition matrices. For the latter the Poisson-type limit is taken in a straightforward way. 
Let us now describe the time-inhomogeneous Markov dynamics $\mathcal{B}_{q, \nu}^{\mathrm{qH}}\left(\tau, \tau^{\prime}\right)$ in terms of generators. Taking the limit $\varepsilon \searrow 0$ in the probabilities (4.2) and using Lemma 4.2 leads to the jump rates

$$
n \cdot \psi_{q, \nu}^{\bullet}\left(x_{n}^{\prime}-x_{n+1}-1 \mid x_{n}-x_{n+1}-1\right)
$$

with which each particle $x_{n}$ jumps to the left into $x_{n}^{\prime} \in\left\{x_{n+1}+1, x_{n+1}+2, \ldots, x_{n}-1\right\}$. The factor $n$ appears from the expansion $r^{n}=(1-\varepsilon)^{n}=1-n \varepsilon+O\left(\varepsilon^{2}\right)$. Denote the Markov generator

with the jump rates (4.4) by $\mathrm{B}_{q, \nu}^{\mathrm{qH}}$. The action of this generator is well-defined because the configurations from $\operatorname{Conf}_{\text {fin }}(\mathbb{Z})$ are densely packed to the left, so only finitely many particles can jump in finite time. Propositions 4.4 and 4.5 then imply that the semigroup and the generator are related as

$$
\mathcal{B}_{q, \nu}^{\mathrm{qH}}\left(\tau, \tau^{\prime}\right)=\exp \left\{\int_{\tau}^{\tau^{\prime}} \mathrm{B}_{q, \nu \mathrm{e}^{-s}}^{\mathrm{qH}} \mathrm{d} s\right\}, \quad \mathrm{B}_{q, \nu}^{\mathrm{qH}}=\left.\frac{\mathrm{d}}{\mathrm{d} \tau^{\prime}}\right|_{\tau^{\prime}=0} \mathcal{B}_{q, \nu}^{\mathrm{qH}}\left(0, \tau^{\prime}\right) .
$$

In words, $\mathcal{B}_{q, \nu}^{\mathrm{qH}}\left(\tau, \tau^{\prime}\right)$ is the Markov transition operator from time $\tau$ to time $\tau^{\prime}$ of a process under which at each time $s$ the jumps are governed by the infinitesimal generator $\mathrm{B}_{q, \nu \mathrm{e}^{-s}}^{\mathrm{qH}}$. We call the process corresponding to $\mathcal{B}_{q, \nu}^{\mathrm{qH}}\left(\tau, \tau^{\prime}\right)$ the backward q-Hahn process.

Remark 4.6. A time- and space-homogeneous version of the backward $q$-Hahn process was considered in [3]. Indeed, the left jumps with rates $\phi_{q, \nu}^{L}\left(x_{n}-x_{n}^{\prime} \mid x_{n}-x_{n+1}-1\right)$ in the $q$-Hahn asymmetric exclusion process from [3] coincide (up to the constant factor $L$ ) with the jumps at rates $\psi_{q, \nu}^{\bullet}\left(x_{n}^{\prime}-x_{n+1}-1 \mid x_{n}-x_{n+1}-1\right)$. However, the spatial inhomogeneity of the backward $q$-Hahn process does not allow to immediately apply the contour integral $q$-moment formulas from [3] in our situation.

\subsection{Action on distributions}

For $\mathrm{t} \in \mathbb{R}_{\geq 0}$ and $\nu \in[0,1)$ denote by $\mathcal{M}_{q, \nu ; \mathrm{t}}^{\mathrm{qH}}$ the time $\mathrm{t}$ distribution of the continuous $q$-Hahn TASEP started from step. The combined results of Sections 4.2 and 4.3 imply the following action of the backward $q$-Hahn process on these distributions:

Theorem 4.7. We have for all $\nu \in[0,1)$ and $\mathrm{t}, \tau \in \mathbb{R}_{\geq 0}$ :

$$
\mathcal{M}_{q, \nu ; \mathrm{t}}^{\mathrm{qH}} \mathcal{B}_{q, \nu}^{\mathrm{qH}}(0, \tau)=\mathcal{M}_{q, \nu \mathrm{e}^{-\tau} ; \mathrm{te}}^{\mathrm{qH}} \text {. }
$$

In words, the time-inhomogeneous backward $q$-Hahn process maps the distribution $\mathcal{M}_{q, \nu ; \mathrm{t}}^{\mathrm{qH}}$ onto the distribution from the same family, but with rescaled parameters $\mathrm{t}$ and $\nu$. See Fig. 6 for an illustration of the action on parameters.

It should be noted that here the forward $q$-Hahn TASEP has homogeneous parameters $\nu_{n} \equiv \nu$, while the construction of the backward process relies on Markov swap operators coming from inhomogeneous parameters $\nu_{n}$.

Proof of Theorem 4.7. Take the continuous time multiparameter $q$-Hahn TASEP from Remark 4.3 and set the parameters to $\nu_{n}=\nu r^{n-1}$, where $\nu \in[0,1)$ and $r \in(0,1)$. After rescaling the continuous time by $\nu$, under this process each particle $x_{n}$ jumps by $j$ at rate $r^{n-1} \psi_{q, \nu r^{n-1}}\left(j \mid x_{n-1}-x_{n}-1\right)$. Denote the distribution of this inhomogeneous process at time $\mathrm{t} \in \mathbb{R}_{\geq 0}$ started from step by $\mathcal{M}_{q, \nu ; \mathrm{t}}^{\mathrm{qH}(r)}$. Clearly,

$$
\lim _{r \rightarrow 1} \mathcal{M}_{q, \nu ; \mathrm{t}}^{\mathrm{qH}(r)}=\mathcal{M}_{q, \nu ; \mathrm{t}}^{\mathrm{qH}} .
$$




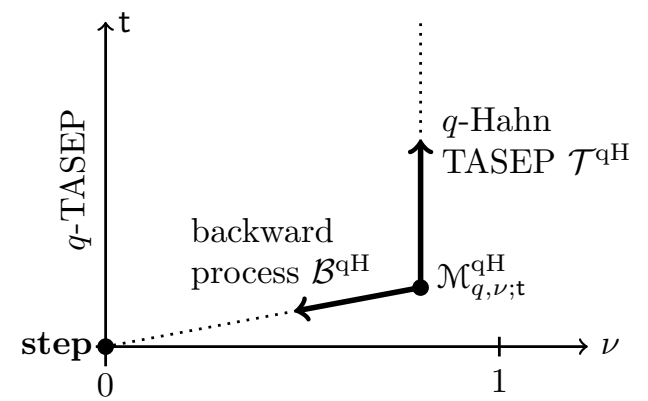

Figure 6. Action of the continuous time $q$-Hahn TASEP and the backward $q$-Hahn process on the measures $\mathcal{M}_{q, \nu ; \mathrm{t}}^{\mathrm{qH}}$ viewed as a two-parameter family depending on $\mathrm{t}$ and $\nu$. The vertical line $\nu=0$ corresponds to distributions of the $q$-TASEP, and on them we obtain a stationary dynamics discussed in Section 5 below.

A suitable modification of Proposition 4.4 applies to this $r$-dependent distribution $\mathcal{M}_{q, \nu ; \mathrm{t}}^{\mathrm{qH}(r)}$, when we take a sequence of discrete Markov backward steps:

$$
\mathcal{M}_{q, \nu ; \mathrm{t}}^{\mathrm{qH}(r)} \mathcal{B}_{q, \nu}^{\mathrm{qH}(r)} \mathcal{B}_{q, r \nu}^{\mathrm{qH}(r)} \mathcal{B}_{q, r^{2} \nu}^{\mathrm{qH}(r)} \cdots \mathcal{B}_{q, r^{k-1} \nu}^{\mathrm{qH}(r)}=\mathcal{M}_{q, r^{k} \nu ; r^{k} \mathrm{t}}^{\mathrm{qH}(r)} .
$$

Indeed, the application of all the operators $\mathcal{B}_{q, r^{i} \nu}^{\mathrm{qH}(r)}, i=0,1, \ldots, k-1$, turns $r^{n-1} \psi_{q, \nu r^{n-1}}$, the jump rate of $x_{n}$, into $r^{k+n-1} \psi_{q, \nu r^{k+n-1}}$. The latter is the same as the old jump rate but with the parameters $(\nu, \mathrm{t})$ multiplied by $r^{k}$. Taking the limit as $r \rightarrow 1$ in (4.5) and using Proposition 4.5 implies the result.

\subsection{Corollary. Mapping TASEP back in time}

By setting $q=\nu=0$ in Theorem 4.7, we recover the main result of the recent paper [41] on the existence of a Markov process which maps the TASEP distributions back in time. Indeed, we have for the rates (4.1):

$$
\psi_{0,0}(j \mid m)=\mathbf{1}_{j=1}, \quad \psi_{0,0}^{\bullet}\left(j^{\prime} \mid m\right)=1,
$$

where $1 \leq j \leq m$ and $0 \leq j^{\prime} \leq m-1$. Moreover, for $q=\nu=0$ the backward continuous time process $\overline{\mathcal{B}_{0,0}^{\mathrm{qH}}}\left(\tau, \tau^{\prime}\right)=\mathcal{B}_{0,0}^{\mathrm{q}} \overline{\mathrm{H}}\left(\tau^{\prime}-\tau\right)$ is time-homogeneous. Under this process, each particle $x_{n}$ independently jumps to the left into one of the holes $\left\{x_{n+1}+1, \ldots, x_{n}-1\right\}$ at rate $n$ per each hole. This dynamics is called the backward Hammersley process in [41].

We see from (4.6) that $\mathcal{M}_{0,0 ; \mathrm{t}}^{\mathrm{qH}}$ is the distribution of the usual TASEP at time $\mathrm{t}$ started from the step initial configuration. Under the action of the backward Hammersley process $\mathcal{B}_{0,0}^{\mathrm{qH}}$ for time $\tau$, the distribution $\mathcal{M}_{0,0 ; \mathrm{t}}^{\mathrm{qH}}$ maps into $\mathcal{M}_{0,0 ; \mathrm{te}^{-\tau}}^{\mathrm{qH}}$. This corollary of Theorem 4.7 is precisely Theorem 1 from [41]. In the latter paper the result was obtained in a completely different way using a well-known connection (e.g., see [11]) of TASEP and Schur processes, which are probability distributions on two-dimensional arrays of interlacing particles. In contrast, here we proved the more general Theorem 4.7 involving only observables of the particle systems in one space dimension, and did not rely on Schur like processes in two space dimensions.

\section{Stationary dynamics on the $q$-TASEP distribution}

When $\nu=0$, the $q$-Hahn TASEP turns into the $q$-TASEP introduced in [7]. We continue working in the continuous time setting as in Section 4. In this section we introduce and study a time-homogeneous, continuous time Markov process which is stationary on the distribution of the $q$-TASEP. 


\section{1 $q$-TASEP and the backward process}

The $q$-TASEP is a continuous time Markov dynamics on $\operatorname{Conf}_{\text {fin }}(\mathbb{Z})$ depending on a single parameter $q$. Under it, each particle $x_{n}(\mathrm{t})$ jumps to the right by one at rate $1-q^{x_{n-1}(\mathrm{t})-x_{n}(\mathrm{t})-1}$ (by agreement, $x_{0} \equiv+\infty$, so the first particle performs the Poisson random walk). When the destination of the jump is occupied, the rate is $1-q^{0}=0$, so the jump is blocked automatically. Denote the infinitesimal Markov generator of the $q$-TASEP by T. For the $q$-TASEP started from the step initial configuration step, let $\mathcal{M}_{q ; \mathrm{t}}^{\mathrm{qT}}$ denote its distribution at time $\mathrm{t} \in \mathbb{R}_{\geq 0}$. See Fig. 7 (jumps to the right) for an illustration.

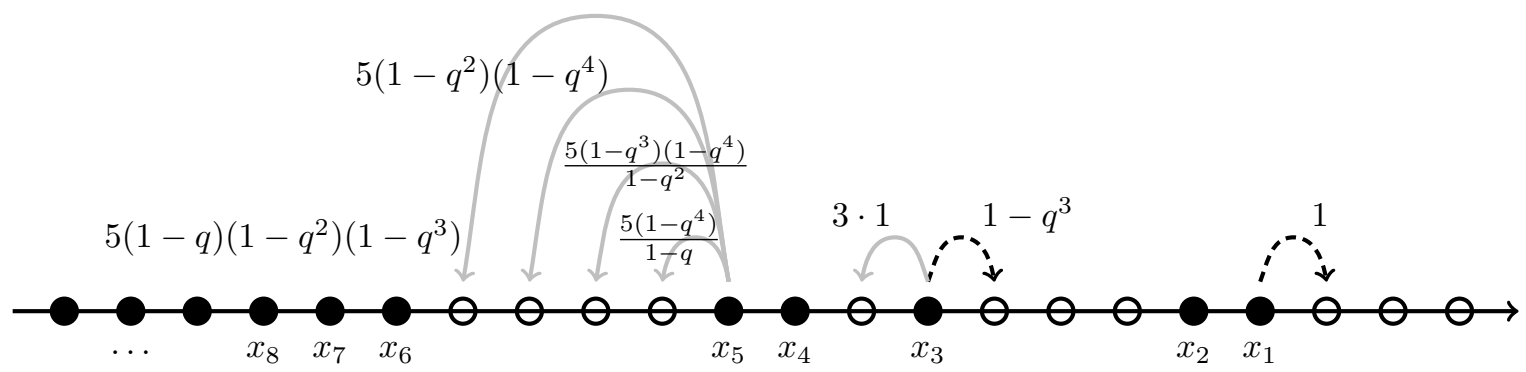

Figure 7. Examples of possible jumps with their rates in the q-TASEP (the dashed arrows to the right) and the backward $q$-TASEP (the gray arrows to the left).

Setting $\nu=0$ in the backward $q$-Hahn process from the previous Section 4 , we arrive at the backward q-TASEP. This specialization in particular makes the backward process timehomogeneous. Under the backward $q$-TASEP process (we denote its continuous time by $\tau$ ), each particle $x_{n}(\tau), n \in \mathbb{Z}_{\geq 1}$, jumps to the left into $x_{n}^{\prime} \in\left\{x_{n+1}(\tau)+1, x_{n+1}(\tau)+2, \ldots, x_{n}(\tau)-1\right\}$ at rate (recall notation (4.1))

$$
n \cdot \psi_{q, 0}^{\bullet}\left(x_{n}^{\prime}-x_{n+1}(\tau)-1 \mid x_{n}(\tau)-x_{n+1}(\tau)-1\right)=\frac{n}{1-q^{x_{n}(\tau)-x_{n}^{\prime}}} \frac{(q ; q)_{x_{n}(\tau)-x_{n+1}(\tau)-1}}{(q ; q)_{x_{n}^{\prime}-x_{n+1}(\tau)-1}} .
$$

Remark 5.1. One can check that

$$
\sum_{x_{n}^{\prime}=x_{n+1}+1}^{x_{n}-1} \psi_{q, 0}^{\bullet}\left(x_{n}^{\prime}-x_{n+1}-1 \mid x_{n}-x_{n+1}-1\right)=x_{n}-x_{n+1}-1,
$$

but we will not use this fact.

Denote the infinitesimal Markov generator of the backward $q$-TASEP by B. See Fig. 7 (jumps to the left) for an illustration.

\subsection{Definition of the stationary dynamics}

Fix the $q$-TASEP time parameter $\mathrm{t} \in \mathbb{R}_{>0}$. Introduce the notation

$$
\mathrm{Q}^{(\mathrm{t})}:=\mathrm{T}+\frac{1}{\mathrm{t}} \mathrm{B} \text {. }
$$

This is an infinitesimal Markov generator of a process under which the particles move to the right according to the $q$-TASEP, and independently move to the left according to the backward $q$-TASEP slowed down by the factor of $t$.

By slightly abusing notation, we denote the continuous time Markov process with the generator $\mathrm{Q}^{(\mathrm{t})}$ by the same letter. We also adopt a convention of using the letter $\tau \in \mathbb{R}_{\geq 0}$ for the continuous time in the process $Q^{(t)}$. Thus, $t$ in $Q^{(t)}$ is a fixed parameter which enters the definition of the process. 
Proposition 5.2. The continuous time Markov process with the generator $\mathrm{Q}^{(\mathrm{t})}$ is well-defined and can start from any configuration $\mathbf{x}(0) \in \operatorname{Conf}_{\text {fin }}(\mathbb{Z})$.

Proof. The only problem in the definition of $Q^{(t)}$ is that it may have infinitely many jumps in finite time. First, observe that the $q$-TASEP is well-defined starting from any configuration $\mathbf{x}(0) \in \operatorname{Conf}_{\text {fin }}(\mathbb{Z})$. Next, under $\mathrm{Q}^{(\mathrm{t})}$ particles go to the right not faster than under the $q$-TASEP. Therefore, with high probability, the random configuration of particles under $Q^{(t)}$ is empty to the right and densely packed to the left outside a bounded region of $\mathbb{Z}$ (the size of the region may depend on the time $\tau$ in $\left.Q^{(t)}\right)$. Because of this, the total jump rate of all particles under $\mathrm{Q}^{(\mathrm{t})}$ is bounded. Therefore, $Q^{(t)}$ does not generate infinitely many jumps in finite time when started from any configuration $\mathbf{x}(0) \in \operatorname{Conf}_{\text {fin }}(\mathbb{Z})$. This completes the proof.

Proposition 5.3. The process $\mathrm{Q}^{(\mathrm{t})}$ preserves the $q$-TASEP distribution $\mathcal{M}_{q ; \mathrm{t}}^{\mathrm{qT}}$.

Proof. By Theorem 4.7, the backward process B, ran for small time $\delta>0$, maps $\mathcal{M}_{q ; \mathrm{t}}^{\mathrm{qT}}$ to $\mathcal{M}_{q ; \mathrm{te}}^{\mathrm{qT}}$. After evolving this distribution under the $q$-TASEP for time $\mathrm{t}-\mathrm{te}^{-\delta}>0$, we get back the original distribution $\mathcal{M}_{q ; \mathrm{t}}^{\mathrm{qT}}$. Differentiating in $\delta$ and sending $\delta \searrow 0$, the infinitesimal Markov generator of the combined dynamics is readily seen to be $t T+B=t Q^{(t)}$. As the factor $t$ by $Q^{(t)}$ simply corresponds to the time scale (of the time variable $\tau$ ), we get the desired statement that the dynamics $\mathrm{Q}^{(\mathrm{t})}$ preserves the measure $\mathcal{M}_{q ; \mathrm{t}}^{\mathrm{qT}}$.

\subsection{Dual process: transient $q$-Boson}

Our aim now is to describe the dual process to $\mathrm{Q}^{(\mathrm{t})}$. by means of the same duality functional $H(\mathbf{x}, \vec{n})=\mathbf{1}_{n_{\ell}>0} \prod_{j=1}^{\ell} q^{x_{n_{j}}+n_{j}}$ (3.15). Recall that $\vec{n} \in \mathbb{W}^{\ell}$ (3.14), and we interpret elements of $\mathbb{W}^{\ell}$ as $\ell$-particle configurations in $\mathbb{Z}_{\geq 0}$.

First consider the individual components $\mathrm{T}$ and $\mathrm{B}$ in $\mathrm{Q}^{(\mathrm{t})}$. The dual process to the $q$-TASEP is known as the stochastic q-Boson particle system [45] (see also [10]). Under this process, particles move in continuous time from site $k$ to $k-1, k \in \mathbb{Z}_{\geq 1}$, independently at different sites. More precisely, if a site $k \in \mathbb{Z}_{\geq 1}$ contains $y_{k}$ particles, then one particle hops from site $k$ to site $k-1$ at rate $1-q^{y_{k}}$. See Fig. 8 (jumps to the left) for an illustration.

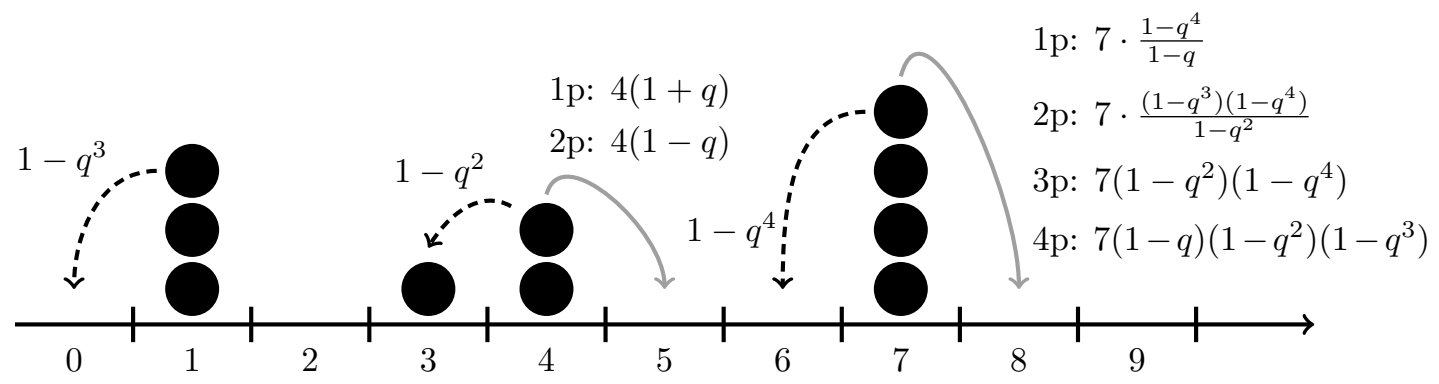

Figure 8. Examples of rates of possible jumps of the stochastic $q$-Boson $\breve{T}$ (dashed jumps to the left) and $\breve{\mathrm{B}}$, the process dual to the backward $q$-TASEP (gray jumps to the right). Each left jump involves only one particle, while right jumps may involve any number of particles in the stack (in the figure, "1p" means that one particle leaves the given stack, and so on).

Denote the infinitesimal Markov generator of the stochastic $q$-Boson process by $\breve{T}$. The duality between $\mathrm{T}$ and $\breve{\mathrm{T}}$ holds in the same sense as in Section 3.4:

Proposition 5.4 ([10]). We have

$$
\mathrm{T} H(\mathbf{x}, \vec{n})=\breve{\mathrm{T}} H(\mathbf{x}, \vec{n})
$$

for any $\mathbf{x} \in \operatorname{Conf}_{\text {fin }}(\mathbb{Z})$ and $\vec{n} \in \mathbb{W}^{\ell}$, where $\mathrm{T}$ acts on $\mathbf{x}$, and $\breve{\mathbf{T}}$ acts on $\vec{n}$. 
Let us now define another continuous time Markov process on $\mathbb{W}^{\ell}$ which will be dual to the backward $q$-TASEP. Under this new process, particles move in continuous time from site $k$ to $k+1, k \in \mathbb{Z}_{\geq 1}$, independently at different sites. More precisely, if a site $k \in \mathbb{Z}_{\geq 0}$ contains $y_{k}$ particles, then the process sends $y_{k}-j$ particles to site $k+1$, where $j \in\left\{0,1, \ldots, y_{k-1}\right\}$, at rate $k \cdot \psi_{q, 0}^{\bullet}\left(j \mid y_{k}\right)$. (In particular, particles cannot leave site 0.) Denote the infinitesimal Markov generator of this dynamics by $\breve{B}$. See Fig. 8 (right jumps) for an illustration.

Proposition 5.5. The Markov generator $\breve{\mathrm{B}}$ is dual to the backward $q$-TASEP generator $\mathrm{B}$ :

$$
\mathrm{B} H(\mathbf{x}, \vec{n})=\breve{\mathrm{B}} H(\mathbf{x}, \vec{n})
$$

for any $\mathbf{x} \in \operatorname{Conf}_{\text {fin }}(\mathbb{Z})$ and $\vec{n} \in \mathbb{W}^{\ell}$, where $\mathrm{B}$ acts on $\mathbf{x}$, and $\breve{\mathrm{B}}$ acts on $\vec{n}$. Consequently, the infinitesimal Markov generator

$$
\breve{\mathrm{Q}}^{(\mathrm{t})}:=\breve{\mathrm{T}}+\frac{1}{\mathrm{t}} \breve{\mathrm{B}}
$$

is dual to $\mathrm{Q}^{(\mathrm{t})}$, the generator of the stationary dynamics on the $q$-TASEP distribution.

Proof. The duality between $B$ and $\breve{B}$ follows from Proposition 3.11 via the continuous time limit described in Section 4. Indeed, the backward $q$-Hahn process is a continuous time limit of the combination of the steps $p_{k}^{\mathrm{qH}}$ applied at each site of the lattice $\mathbb{Z}$. The dual process $\breve{\mathrm{B}}$ is the limit of the same type of the combination of the dual steps $\breve{p}_{k}^{\mathrm{qH}}$, together with the degeneration $\nu=0$. This implies the duality of $\mathrm{B}$ and $\breve{\mathrm{B}}$. The claim about the duality of $\mathrm{Q}^{(\mathrm{t})}$ and $\breve{\mathrm{Q}}^{(\mathrm{t})}$ follows by linearity.

Because the rates of the right jumps under $\breve{Q}^{(t)}$ grow as the particles of $\vec{n}$ get farther to the right, the process $\breve{Q}^{(t)}$ is transient (except the absorption at $n_{\ell}=0$ ), see the proof of Lemma 5.9 below for details. For this reason we call $\breve{Q}^{(t)}$ the transient stochastic $q$-Boson particle system on $\mathbb{W}^{\ell}$ (transient $q$-Boson, for short). Let us record the duality between it and $\mathrm{Q}^{(\mathrm{t})}$ in terms of expectations:

Corollary 5.6. Fix $\mathrm{t} \in \mathbb{R}_{>0}$ and $\ell \in \mathbb{Z}_{>1}$. Take any $\mathrm{x}^{0} \in \operatorname{Conf}_{\text {fin }}(\mathbb{Z})$, and any $\vec{n}^{0} \in \mathbb{W}^{\ell}$. Let $\{\mathbf{x}(\tau)\}_{\tau \in \mathbb{R}_{\geq 0}}$ be the process on $\operatorname{Conf}_{\text {fin }}(\mathbb{Z})$ with generator $\mathrm{Q}^{(\mathrm{t})}$ started from $\mathbf{x}^{0}$, and $\{\vec{n}(\tau)\}_{\tau \in \mathbb{R}_{\geq 0}}$ be the process on $\mathbb{W}^{\ell}$ with generator $\breve{Q}^{(\mathrm{t})}$ started from $\vec{n}^{0}$. Then for any $\tau \in \mathbb{R}_{\geq 0}$ we have

$$
\mathbb{E}_{\mathbf{x}(0)=\mathbf{x}^{0}}^{\text {stat }(\mathrm{t})} H\left(\mathbf{x}(\tau), \vec{n}^{0}\right)=\mathbb{E}_{\vec{n}(0)=\vec{n}^{0}}^{\text {trqBoson }(\mathrm{t})} H\left(\mathbf{x}^{0}, \vec{n}(\tau)\right) .
$$

The example of this duality statement (and further discussion towards the results of the next Section 5.4) in the simplest case $\ell=1$ may be found in Section 1.4 in Introduction.

\subsection{Convergence to the stationary distribution}

In this section we use duality to prove the following result:

Theorem 5.7. Fix $\mathrm{t} \in \mathbb{R}_{>0}$. For any initial configuration $\mathrm{x}(0) \in \operatorname{Conf}_{\text {fin }}(\mathbb{Z})$ the Markov process $\mathbf{x}(\tau)$ with the generator $\mathrm{Q}^{(\mathrm{t})}$ converges, as $\tau \rightarrow+\infty$, to the stationary distribution $\mathcal{M}_{q ; \mathrm{t}}^{\mathrm{qT}}$ (in the sense of joint distributions of arbitrary finite subcollections of particles).

The proof of Theorem 5.7 occupies the rest of this section.

Fix any $\ell \geq 1$ and $\vec{m} \in \mathbb{W}^{\ell}$. Let $\vec{n}(\tau)$ be the transient $q$-Boson $\breve{Q}^{(\mathrm{t})}$ started from $\vec{m}$. Denote the survival probability of the transient $q$-Boson till time $\tau$ by

$$
S_{\tau}(\vec{m}):=\mathbb{P}\left(n_{\ell}(\tau)>0 \mid \vec{n}(0)=\vec{m}\right) .
$$

Note that if $m_{\ell}=0$, we automatically have $S_{\tau}(\vec{m})=0$ for all $\tau$. 
Lemma 5.8. For any $\vec{m} \in \mathbb{W} \ell$, the asymptotic survival probability

$$
S(\vec{m}):=\lim _{\tau \rightarrow+\infty} S_{\tau}(\vec{m})
$$

exists.

Proof. We have

$$
S_{\tau}(\vec{m})=\mathbb{P}\left(n_{\ell}(\tau)>0 \mid \vec{n}(0)=\vec{m}\right)=\mathbb{P}\left(n_{\ell}(s)>0 \text { for all } s \in[0, \tau] \mid \vec{n}(0)=\vec{m}\right)
$$

because once $n_{\ell}$ reaches 0 , it can never become positive again. Therefore, the quantities $S_{\tau}(\vec{m})$ decrease in $\tau$ due to monotonicity in $\tau$ of the events $\left\{n_{\ell}(s)>0\right.$ for all $\left.s \in[0, \tau]\right\}$. Because $S_{\tau}(\vec{m})$ 's are nonnegative, the limit exists.

Observe that $H$ (step, $\vec{m})=\mathbf{1}_{m_{\ell}>0}$. Therefore, for the stationary process $\mathbf{x}(\tau)$ started from step we have $\mathbb{E}_{\text {step }}^{\text {stat }(\mathrm{t})} \prod_{j=1}^{\ell} q^{x_{m_{j}}(\tau)+m_{j}}=S_{\tau}(\vec{m})$ for all $\vec{m} \in \mathbb{W}^{\ell}$ (in particular, if $m_{\ell}=0$ then both sides are zero). For other initial conditions for $\mathrm{Q}^{(\mathrm{t})}$ this identity does not hold for finite time $\tau$, but it still holds asymptotically:

Lemma 5.9. For any $\vec{m} \in \mathbb{W}^{\ell}$ and any initial data $\mathbf{x}^{0} \in \operatorname{Conf}_{\text {fin }}(\mathbb{Z})$ for the stationary process $\mathrm{Q}^{(\mathrm{t})}$, we have

$$
\lim _{\tau \rightarrow+\infty} \mathbb{E}_{\mathbf{x}(0)=\mathbf{x}^{0}}^{\text {stat }(\mathrm{t})} \prod_{j=1}^{\ell} q^{x_{m_{j}}(\tau)+m_{j}}=S(\vec{m}),
$$

where $S(\vec{m})$ is the asymptotic survival probability of the transient q-Boson.

Proof. If $m_{\ell}=0$, then both sides of (5.3) are zero, so it suffices to assume that $m_{\ell}>0$. By duality (Corollary 5.6), the left-hand side of (5.3) is equal to

$$
\lim _{\tau \rightarrow+\infty} \mathbb{E}_{\vec{n}(0)=\vec{m}}^{\operatorname{trqBoson}(\mathrm{t})}\left(\mathbf{1}_{n_{\ell}(\tau)>0} \prod_{j=1}^{\ell} q^{x_{n_{j}(\tau)}^{0}+n_{j}(\tau)}\right) .
$$

Here $x_{n}^{0}$ are the particle coordinates under the initial data $\mathbf{x}^{0}$.

First, we show that the Markov process $\vec{n}(\tau)$ conditioned to stay in the region $\left\{n_{\ell} \geq 1\right\}$ is transient. Observe that we can couple the $\ell$-particle process $\vec{n}(\tau)$ restricted to this region with a single-particle process $Y(\tau)$ on $\mathbb{Z}_{\geq 1}$ with jump rates

$$
\operatorname{rate}_{Y}(k+1 \rightarrow k)=1-q^{\ell}, \quad \operatorname{rate}_{Y}(k \rightarrow k+1)=\frac{k}{\mathrm{t}}\left(\min _{j, r: 0 \leq j<r \leq \ell} \psi_{q, 0}^{\bullet}(j \mid r)\right)>0, \quad k \geq 1 .
$$

The coupling is such that $Y(0)=n_{\ell}(0)$, all left jumps of $n_{\ell}$ force $Y$ to jump to the left, and all right jumps of $Y$ force $n_{\ell}$ to jump to the right. This implies that under this coupling we have $Y(\tau) \leq n_{\ell}(\tau)$ for all $\tau>0$.

The process $Y(\tau)$ on $\mathbb{Z}_{\geq 1}$ is a standard example of a transient Markov process: it eventually (with probability 1 ) reaches the part $\{A, A+1, \ldots\} \subset \mathbb{Z}_{\geq 1}$ (where $A$ depends only on $\ell$ and $\mathrm{t}$ ) where the average drift to the right is bounded away from zero. With positive probability $Y(\tau)$ then never comes back from $\{A, A+1, \ldots\}$ to the neighborhood of zero, and thus is transient.

Using the transience, we can lower bound $S(\vec{m})$ by the (positive) probability of the event that: (1) if there were any particles at site 1 at time $\tau=0$, then all these particles leave 1 by right jumps; (2) after that, $n_{\ell}(\tau)$ never comes back to 1 (and hence all other particles of $\vec{n}(\tau)$ also never come back to 1). This implies that $S(\vec{m})>0$. 
Now denote by $\mathcal{S}(\vec{m})$ the event that $n_{\ell}(\tau)>0$ for all $\tau$ conditioned on $\vec{n}(0)=\vec{m}$. Thus, $\mathbb{P}(\mathcal{S}(\vec{m}))=S(\vec{m})>0$. We have for the expectation in $(5.4)$ :

$$
\begin{gathered}
\mathbb{E}_{\vec{n}(0)=\vec{m}}^{\operatorname{trqBoson}(\mathrm{t})}\left(\mathbf{1}_{n_{\ell}(\tau)>0} \prod_{j=1}^{\ell} q^{x_{n_{j}(\tau)}^{0}+n_{j}(\tau)}\right)=S(\vec{m}) \mathbb{E}_{\vec{n}(0)=\vec{m}}^{\operatorname{trqBoson}(\mathrm{t})}\left(\prod_{j=1}^{\ell} q^{x_{n_{j}(\tau)}^{0}+n_{j}(\tau)} \mid \mathcal{S}(\vec{m})\right) \\
+(1-S(\vec{m})) \mathbb{E}_{\vec{n}(0)=\vec{m}}^{\operatorname{trqBoson}(\mathrm{t})}\left(\mathbf{1}_{n_{\ell}(\tau)>0} \prod_{j=1}^{\ell} q^{x_{n_{j}(\tau)}^{0}+n_{j}(\tau)} \mid \mathcal{S}(\vec{m})^{c}\right) .
\end{gathered}
$$

The second summand goes to zero as $\tau \rightarrow+\infty$, since inside the event $\mathcal{S}(\vec{m})^{c}$, we have $n_{\ell}(s)=0$ for all $s \in\left(s_{0},+\infty\right)$ (where $s_{0}$ is random but finite). In the first summand, observe that conditioned on the asymptotic survival $\mathcal{S}(\vec{m})$, we have almost surely due to transience that $n_{j}(\tau) \rightarrow+\infty, \tau \rightarrow+\infty$, for all $j=1, \ldots, \ell$. Because the initial configuration $\mathbf{x}^{0} \in \operatorname{Conf}_{\text {fin }}(\mathbb{Z})$ is densely packed to the left, we thus almost surely have $q^{x_{n_{j}(\tau)}^{0}+n_{j}(\tau)} \rightarrow 1$ for all $j$ as $\tau \rightarrow+\infty$. Therefore, the expectation of the product in the first summand tends to 1 , and we see that (5.4) is equal to $S(\vec{m})$, as desired.

The asymptotic survival probabilities $S(\vec{m})$ satisfy certain normalization at infinity:

Lemma 5.10. Fix $\ell$. For any $\varepsilon>0$ there exists $R=R(\ell, \varepsilon) \in \mathbb{Z}_{\geq 1}$ such that for all $\vec{m} \in \mathbb{W}^{\ell}$ with $m_{1}>R$ we have

$$
\left|S\left(m_{1}, m_{2}, \ldots, m_{\ell}\right)-S\left(m_{2}, \ldots, m_{\ell}\right)\right|<\varepsilon,
$$

where $S\left(m_{2}, \ldots, m_{\ell}\right)$ is the survival probability of the transient $q$-Boson on $\mathbb{W}^{\ell-1}$. If $\ell=1$, then $S\left(m_{2}, \ldots, m_{\ell}\right)=1$ by agreement.

Proof. We can assume that $m_{\ell}>0$, otherwise both expressions $S(\cdot)$ in the claim are zero. The desired statement follows from the transience of the process $\vec{n}(\tau)$ (with generator $\breve{Q}^{(\mathrm{t})}$ ) as in the proof of the previous Lemma 5.9. Namely, we can lower bound the jump rate of $n_{1}(\tau)$ to the right from a site $k \in \mathbb{Z}_{\geq 1}$ by const. $k$. Let $\vec{n}(\tau)$ start from $\vec{m}$. If $m_{1}>R$ is large, the probability that the first particle $n_{1}(\tau)$ ever returns to the $R / 2$-neighborhood of zero is close to zero. Thus, the probability $S(\vec{m})$ that the process $\vec{n}(\tau)$ started from $\vec{m}$ survives and runs off to infinity is close to the asymptotic survival probability of the process on $\mathbb{W}^{\ell-1}$ with one less particle and started from $\left(m_{2}, \ldots, m_{\ell}\right)$. In the special case $\ell=1$, the claim reads $S\left(m_{1}\right) \rightarrow 1$ as $m_{1} \rightarrow+\infty$, which clearly holds. This implies the claim.

Remark 5.11. If under the conditions of Lemma 5.10 the second coordinate $m_{2}$ is also very large, then one can similarly show that both $S\left(m_{1}, m_{2}, m_{3}, \ldots, m_{\ell}\right)$ and $S\left(m_{2}, m_{3}, \ldots, m_{\ell}\right)$ are close to $S\left(m_{3}, \ldots, m_{\ell}\right)$, and thus close to each other. Hence an analogue of Lemma 5.10 for a number of first coordinates $m_{1}, \ldots, m_{j}$ being large also holds.

To finish the proof of Theorem 5.7 it remains to show that

$$
S(\vec{m})=\mathbb{E}_{\text {step }}^{\mathrm{qT}} \prod_{j=1}^{\ell} q^{x_{m_{j}}(\mathrm{t})+m_{j}}
$$

for all $\ell$ and $\vec{m} \in \mathbb{W}^{\ell}$. Here the quantity in the left-hand side is the long time limit of the $q$-moment of $\mathrm{Q}^{(\mathrm{t})}$, and the right-hand side is the $q$-moment of the $q$-TASEP. This suffices since in our situation the $q$-moments uniquely characterize the distribution. We will establish (5.5) by showing that both sides satisfy the same equations (harmonicity with respect to the transient $q$-Boson) plus normalization at infinity which uniquely determine the function. 
Lemma 5.12. As function of $\vec{m}$, the survival probabilities $S(\vec{m})$ are harmonic for the transient $q$-Boson, that is,

$$
\left(\breve{\mathrm{Q}}^{(\mathrm{t})} S\right)(\vec{m})=\sum_{\vec{m}^{\prime}} \breve{\mathrm{Q}}^{(\mathrm{t})}\left(\vec{m}, \vec{m}^{\prime}\right) S\left(\vec{m}^{\prime}\right)=0 \quad \text { for all } \vec{m} \in \mathbb{W}^{\ell}
$$

Here the first identity is simply the expression for the action of the generator on a function, and the claim is that this action gives identical 0.

Proof. The argument is rather standard. Consider the evolution of the process $\vec{n}(\cdot)$ started from $\vec{m}$ during short time $\mathrm{d} \tau$. Conditioned that the process stepped into $\vec{m}^{\prime}$ (which happens with probability $\left.\breve{Q}^{(\mathrm{t})}\left(\vec{m}, \vec{m}^{\prime}\right) \mathrm{d} \tau\right)$, the survival probability is then $S\left(\vec{m}^{\prime}\right)$. With the complementary probability $1-\sum_{\vec{m}^{\prime}: \vec{m}^{\prime} \neq \vec{m}} \breve{\mathrm{Q}}^{(\mathrm{t})}\left(\vec{m}, \vec{m}^{\prime}\right) \mathrm{d} \tau=1+\breve{\mathrm{Q}}^{(\mathrm{t})}(\vec{m}, \vec{m}) \mathrm{d} \tau$, the process did not leave $\vec{m}$, and the survival probability did not change. Therefore,

$$
S(\vec{m})=S(\vec{m})+\mathrm{d} \tau \sum_{\vec{m}^{\prime}} \breve{\mathrm{Q}}^{(\mathrm{t})}\left(\vec{m}, \vec{m}^{\prime}\right) S\left(\vec{m}^{\prime}\right) .
$$

Taking the coefficient by $\mathrm{d} \tau$ leads to the desired identity.

Lemma 5.13. Harmonicity condition (5.6) together with Lemma 5.10 (normalization at infinity) and the condition that $S(\vec{m})=0$ whenever $m_{\ell}=0$ uniquely determine the function $S(\vec{m})$, $\vec{m} \in \mathbb{W}^{\ell}$.

Proof. Assume that $G_{\ell}(\vec{m})$, where $\ell=1,2, \ldots$ and $\vec{m} \in \mathbb{W}^{\ell}$, is a family of harmonic functions satisfying normalization at infinity as in Lemma 5.10, that is, for any $\varepsilon>0$ there exists $R$ such that for all $\vec{m} \in \mathbb{W}^{\ell}$ with $m_{1}>R$ we have

$$
\left|G_{\ell}\left(m_{1}, m_{2}, \ldots, m_{\ell}\right)-G_{\ell-1}\left(m_{2}, \ldots, m_{\ell}\right)\right|<\varepsilon .
$$

Moreover, we assume that $G_{\ell}(\vec{m})=0$ whenever $m_{\ell}=0$. We will argue by induction on $\ell$ and show that $G_{\ell}(\vec{m})$ is equal to $S(\vec{m})$, the asymptotic survival probability of the transient $q$-Boson on $\mathbb{W}^{\ell}$.

For $\ell=1$, it is straightforward to see that the space of harmonic functions vanishing at 0 is one-dimensional. In this case the normalization at infinity is the single condition $G_{1}\left(m_{1}\right) \rightarrow 1$ as $m_{1} \rightarrow+\infty$, which determines the harmonic function uniquely.

Next, observe that because the function $G_{\ell}$ is harmonic, it satisfies the following averaging property:

$$
G_{\ell}(\vec{m})=\mathbb{E}_{\vec{n}(0)=\vec{m}}^{\operatorname{trqBoson}(\mathrm{t})} G_{\ell}(\vec{n}(\tau)),
$$

where $\tau$ is arbitrary.

Assume that $m_{\ell}>0$ (this does not restrict the generality). For $R \in \mathbb{Z}_{\geq 1}$, take the stopping time

$$
T_{R}:=\inf \left\{\tau \geq 0: n_{\ell}(\tau)=0 \text { or } n_{1}(\tau)=R\right\},
$$

where the process $\vec{n}(\tau)$ starts from $\vec{m}$. This stopping time is almost surely bounded and has finite expectation. Then

$$
G_{\ell}(\vec{m})=\mathbb{E}_{\vec{n}(0)=\vec{m}}^{\mathrm{trqBoson}(\mathrm{t})} G_{\ell}\left(\vec{n}\left(T_{R}\right)\right)=\mathbb{E}_{\vec{n}(0)=\vec{m}}^{\operatorname{trqBoson}(\mathrm{t})}\left\{G_{\ell}\left(R, n_{2}\left(T_{R}\right), \ldots, n_{\ell}\left(T_{R}\right)\right) \mathbf{1}_{n_{1}\left(T_{R}\right)=R}\right\} .
$$

The first equality above follows from the optional stopping theorem, and the second one is the splitting into two cases, $n_{1}\left(T_{R}\right)=R$ or $n_{\ell}\left(T_{R}\right)=0$. In the latter case the function $G_{\ell}$ vanishes by our assumptions. 
Take any $\varepsilon>0$ and choose $R$ such that (5.7) holds. Thus, by the normalization at infinity, we have

$$
\left|\operatorname{RHS}(5.8)-\mathbb{E}_{\vec{n}(0)=\vec{m}}^{\operatorname{trqBoson}(\mathrm{t})}\left\{S\left(n_{2}\left(T_{R}\right), \ldots, n_{\ell}\left(T_{R}\right)\right) \mathbf{1}_{n_{1}\left(T_{R}\right)=R}\right\}\right|<\varepsilon,
$$

where we have replaced $G_{\ell-1}$ by $S$ using the induction hypothesis. Since $S$ satisfies the same conditions (harmonicity, normalization at infinity, and vanishing when $m_{\ell}=0$ ) as the family $G_{\ell}$, identity (5.8) is also valid for $S$. Thus,

$$
\left|\mathbb{E}_{\vec{n}(0)=\vec{m}}^{\mathrm{trqBoson}(\mathrm{t})}\left\{S\left(n_{2}\left(T_{R}\right), \ldots, n_{\ell}\left(T_{R}\right)\right) \mathbf{1}_{n_{1}\left(T_{R}\right)=R}\right\}-S\left(m_{1}, \ldots, m_{\ell}\right)\right|<\varepsilon,
$$

which means that $G_{\ell}\left(m_{1}, \ldots, m_{\ell}\right)$ is $\varepsilon$-close to $S\left(m_{1}, \ldots, m_{\ell}\right)$. This completes the proof.

Lemma 5.14. The q-moments of the q-TASEP in the right-hand side of (5.5) satisfy all the conditions listed in the previous Lemma 5.13.

Proof. The harmonicity of the $q$-moments follows from the fact that the $q$-TASEP distribution $\mathcal{M}_{q ; \mathrm{t}}^{\mathrm{qT}}$ is stationary under $\mathrm{Q}^{(\mathrm{t})}$ (Proposition 5.3), together with duality between $\mathrm{Q}^{(\mathrm{t})}$ and the transient $q$-Boson (Corollary 5.6).

The normalization at infinity follows from the fact that the $q$-TASEP started from step lives on $\operatorname{Conf}_{\text {fin }}(\mathbb{Z})$, so for any $\mathrm{t} \in \mathbb{R}_{\geq 0}$ we almost surely have $x_{m}(\mathrm{t})+m \rightarrow 0$ as $m \rightarrow+\infty$.

The fact that the $q$-moments vanish when $n_{\ell}=0$ follows from the agreement that $x_{0}=+\infty$.

Combining all the lemmas in this section, we get the desired Theorem 5.7.

\section{Beta polymer}

The $q$-Hahn TASEP has a remarkable degeneration - the beta polymer model introduced in [4]. This model is also related to a random walk in dynamic beta random environment, but here we will formulate everything only in terms of the polymer model. In this section we present Markov swap operators for the multiparameter beta polymer. The swap operators can be realized as certain additional layers in the strict-weak lattice on which the beta polymer is defined.

\subsection{Multiparameter beta polymer and its joint moments}

Take parameters $\gamma>0$ and $v_{n}>0, t, n \in \mathbb{Z}_{\geq 1}$, such that

$$
v_{n}-\gamma>0 \text { for all } n, \quad v_{i}-v_{j} \notin \mathbb{Z} \text { for all } i, j .
$$

Let $B_{t, n} \sim \operatorname{Beta}\left(v_{n}-\gamma, \gamma\right)$ be independent beta distributed random variables. Here by the beta distribution $\operatorname{Beta}(\alpha, \beta)$ we mean the one with the density

$$
\frac{\Gamma(\alpha+\beta)}{\Gamma(\alpha) \Gamma(\beta)} x^{\alpha-1}(1-x)^{\beta-1}, \quad x \in[0,1] .
$$

The (inhomogeneous) beta polymer $\{Z(t, n)\}_{t, n \in \mathbb{Z}_{\geq 1}}$ is a collection of random variables satisfying the random recursion

$$
\begin{aligned}
Z(t, n) & =B_{t, n} Z(t-1, n)+\left(1-B_{t, n}\right) Z(t-1, n-1), \\
Z(t, 1) & =B_{t, 1} Z(t-1,1),
\end{aligned}
$$

with the initial condition $Z(0, n)=1$ for all $n \in \mathbb{Z}_{\geq 1}$. See Fig. 9 for a graphical interpretation of the beta polymer as a point-to-line partition function on the strict-weak lattice. 


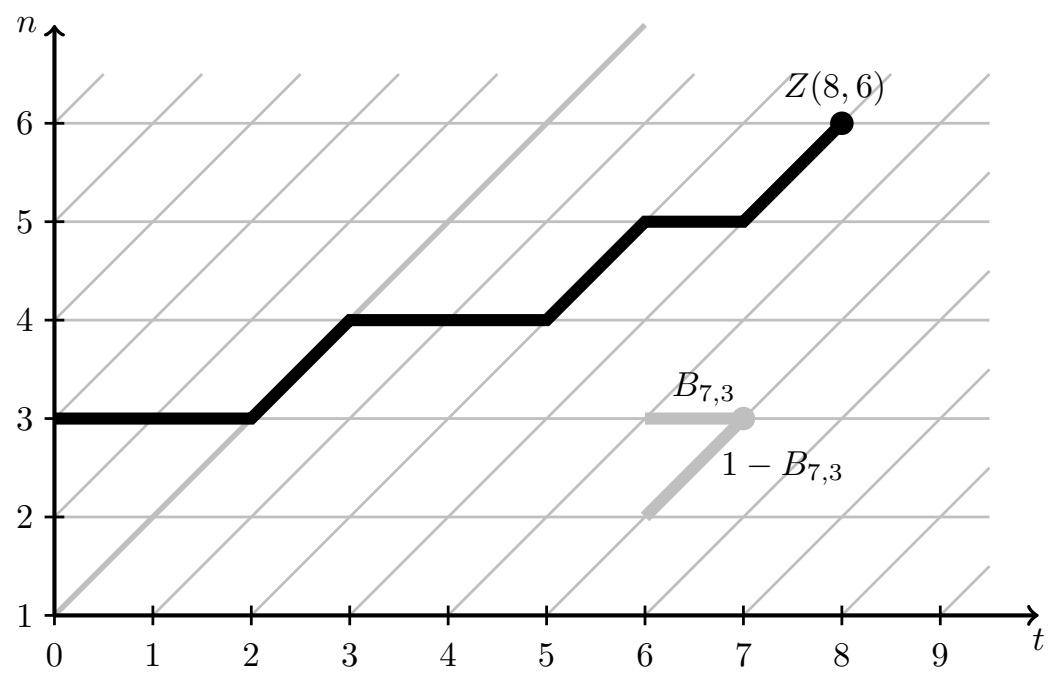

Figure 9. The beta polymer partition function $Z(t, n)$ is the sum of weights of all strict-weak paths from the line $\{0\} \times \mathbb{Z}_{\geq 1}$ to $(t, n)$, where the weights of the horizontal and the diagonal edges are $B \ldots$ and $1-B \ldots$, respectively. The weight of a path is defined as the product of its edge weights. The initial condition along the left boundary is $Z(0, n)=1$ for all $n$, so $Z(t, n)=1$ above the diagonal (i.e., for $n>t$ ).

The homogeneous version of the beta polymer was studied in [4] as a scaling limit $q \rightarrow 1$ of the $q$-Hahn TASEP. The multiparameter generalization is obtained through the same limit from our model described in Section 3.

The random variables $Z(t, n)$ are between 0 and $1 .^{5}$ Because of this, the joint distribution of the beta polymer random variables $\{Z(t, n)\}_{n \in \mathbb{Z}_{\geq 1}}$ (for every fixed $t$ ) is determined by the joint moments. These moments have the following form:

Proposition 6.1. For each $t \in \mathbb{Z}_{\geq 1}$ and any $n_{1} \geq \cdots \geq n_{k} \geq 1$ we have

$$
\begin{aligned}
\mathbb{E}^{\operatorname{beta}(\boldsymbol{v})}\left(Z\left(t, n_{1}\right) \cdots Z\left(t, n_{k}\right)\right) & \\
= & \frac{1}{(2 \pi i)^{k}} \oint \cdots \oint \prod_{1 \leq A<B \leq k} \frac{z_{A}-z_{B}}{z_{A}-z_{B}-1} \prod_{j=1}^{k}\left(\prod_{i=1}^{n_{j}} \frac{z_{j}}{z_{j}-v_{i}}\right)\left(\frac{z_{j}-\gamma}{z_{j}}\right)^{t} \frac{\mathrm{d} z_{j}}{z_{j}} .
\end{aligned}
$$

The integration contours are around $\left\{v_{i}\right\}$, do not encircle 0 , and the contour for $z_{j}$ contains the contour for $z_{j+1}+1, j=1, \ldots, k-1$.

Proof. For $v_{i} \equiv v$, this formula is a simple change of variables from [4, Proposition 2.11], where $\mu=v-\gamma$. Its generalization with different $v_{i}$ 's is obtained by duality and coordinate Bethe ansatz in the same manner as in [4] by checking that the right-hand side of (6.1) satisfies the free evolution equations, boundary conditions, and the initial condition at $t=0$.

For future use, let us recall here the free evolution equations and the boundary conditions satisfied by the right-hand side of (6.1). Denote this right-hand side by $f\left(t ; n_{1}, \ldots, n_{k}\right)$. Then it satisfies the two-body boundary conditions

$$
\begin{aligned}
& f\left(t ; n_{1}, \ldots, n_{i}-1, n_{i+1}-1, \ldots, n_{k}\right)+\left(v_{n_{i}}-1\right) f\left(t ; n_{1}, \ldots, n_{i}, n_{i+1}-1, \ldots, n_{k}\right) \\
& \quad+f\left(t ; n_{1}, \ldots, n_{i}, n_{i+1}, \ldots, n_{k}\right)-\left(v_{n_{i}}+1\right) f\left(t ; n_{1}, \ldots, n_{i}-1, n_{i+1}, \ldots, n_{k}\right)=0
\end{aligned}
$$

for all $\vec{n} \in \mathbb{Z}^{k}$ such that for some $i \in\{1, \ldots, k\}, n_{i}=n_{i+1}$. This is checked similarly to the homogeneous case [4, Section 4.1], because the inhomogeneity parameters $v_{n_{i}}$ are the same

\footnotetext{
${ }^{5}$ Moreover, one can check that for fixed $t$ they are ordered as $1 \geq Z(t, 1) \geq Z(t, 2) \geq Z(t, 3) \geq \cdots$.
} 
for each cluster of equal $n_{j}$ 's (a similar effect is observed in the proof of Lemma 3.7 where only one of the parameters, $\nu_{n}$, plays an essential role).

The free evolution equations satisfied by $f(t ; \vec{n})$ are

$$
f(t+1 ; \vec{n})=\prod_{i=1}^{k}\left[\nabla_{\gamma / v_{n_{i}}}^{\text {beta }}\right]_{i} f(t ; \vec{n})
$$

where $\nabla_{p}^{\text {beta }} g(n):=p g(n-1)+(1-p) g(n)$, and the operator $\left[\nabla_{\gamma / v_{n_{i}}}^{\text {beta }}\right]_{i}$ is applied in the $i$-th variable $n_{i}$.

Remark 6.2. Similarly to Remark 3.5, one can generalize the beta polymer model so that the parameter $\gamma$ depends on $t$. The moment formula (6.1) and our main result (Theorem 6.4 below) would continue to hold with straightforward modifications. For simplicity, everywhere below we take $\gamma$ independent of $t$.

Proposition 6.3. The multiparameter beta polymer, viewed as a stochastic particle system $Z(t, \cdot)$ on $[0,1]$ with time $t \in \mathbb{Z}_{\geq 1}$, is parameter-symmetric in the sense of Definition 2.1. Moreover, the distribution of each $Z(t, n)$ depends on the parameters $v_{1}, \ldots, v_{n}$ in a symmetric way.

\subsection{Swap operator for the beta polymer}

Let $\left\{Z^{\boldsymbol{v}}(t, n)\right\}_{t, n \in \mathbb{Z}_{\geq 1}}$ be the beta polymer with parameters $\boldsymbol{v}=\left\{\boldsymbol{v}_{1}, \boldsymbol{v}_{2}, \ldots\right\}$. Recall that by $s_{n}$ we denote the elementary transposition $(n, n+1)$. The next statement presents the swap operator interchanging $v_{n} \leftrightarrow v_{n+1}$.

Theorem 6.4. Fix $t, n \in \mathbb{Z}_{\geq 1}$ and assume that $v_{n}<v_{n+1}$. Let $\tilde{B} \sim \operatorname{Beta}\left(v_{n+1}-v_{n}, v_{n}\right)$ be a new beta random variable independent of the environment $\left\{B_{t, n}\right\}$ (and hence of the beta polymer). Then we have equality in distribution

$$
\begin{aligned}
\left(Z^{\boldsymbol{v}}(t, 1), \ldots, Z^{\boldsymbol{v}}(t, n-1),\right. & \left.\tilde{B} Z^{\boldsymbol{v}}(t, n+1)+(1-\tilde{B}) Z^{\boldsymbol{v}}(t, n), Z^{\boldsymbol{v}}(t, n+1)\right) \\
& \stackrel{d}{=}\left(Z^{s_{n} \boldsymbol{v}}(t, 1), \ldots, Z^{s_{n} \boldsymbol{v}}(t, n-1), Z^{s_{n} \boldsymbol{v}}(t, n), Z^{s_{n} \boldsymbol{v}}(t, n+1)\right) .
\end{aligned}
$$

In other words, when $v_{n}<v_{n+1}$, the beta polymer admits a Markov swap operator $p_{n}^{\text {beta }}$ (in the sense of Definition 2.5) which acts by splitting the segment $\left[Z^{v}(t, n+1), Z^{v}(t, n)\right] \subset[0,1]$ as $1-\tilde{B}: \tilde{B}$, and replacing $Z^{\boldsymbol{v}}(t, n)$ by the separating point.

Proof of Theorem 6.4. The proof is similar to the case of the $q$-Hahn TASEP given in Section 3.3. Here we briefly outline the main computations. We use the notation (3.8) which we reproduce here for convenience:

$$
\vec{n}=\left(n_{1}, \ldots, n_{k}\right)=(m_{1}, \ldots, m_{\ell}, \underbrace{n+1, \ldots, n+1}_{a}, \underbrace{n, \ldots, n}_{b}, m_{1}^{\prime}, \ldots, m_{\ell^{\prime}}^{\prime}) .
$$

Applying $p_{n}^{\text {beta }}$ to the moment formula, we will compute moments of the form

$$
\mathbb{E}^{\operatorname{beta}(\boldsymbol{v})}\left\{(\tilde{B} Z(t, n+1)+(1-\tilde{B}) Z(t, n))^{b} \prod_{\substack{j=1 \\ n_{j} \neq n}}^{k} Z\left(t, n_{j}\right)\right\} .
$$

Expanding $(\tilde{B} Z(t, n+1)+(1-\tilde{B}) Z(t, n))^{b}$ and using the independence of $\tilde{B}$ from the polymer, we have (see, for example, [4, Lemma 4.1] for the moments of the beta distribution)

$$
(6.4)=\sum_{r=0}^{b}\left(\begin{array}{l}
b \\
r
\end{array}\right) \frac{\left(v_{n}\right)_{r}\left(v_{n+1}-v_{n}\right)_{b-r}}{\left(v_{n+1}\right)_{b}} \mathbb{E}^{\operatorname{beta}(v)}\left\{Z(t, n)^{r} Z(t, n+1)^{a+b-r} \prod_{\substack{j=1 \\
n_{j} \neq n, n+1}}^{k} Z\left(t, n_{j}\right)\right\},
$$


where $(\alpha)_{k}:=\alpha(\alpha+1) \cdots(\alpha+k-1)$ is the Pochhammer symbol. Denote the expectation in (6.5) by

$$
g(t ; m_{1}, \ldots, m_{\ell}, \underbrace{n+1, \ldots, n+1}_{a+b-r}, \underbrace{n, \ldots, n}_{r}, m_{1}^{\prime}, \ldots, m_{\ell^{\prime}}^{\prime}) .
$$

This expectation satisfies the two-body boundary conditions (6.2) with the parameter $v=v_{n+1}$. Therefore, using the argument from [4, Section 4] (a statement parallel to the $q$-Hahn TASEP's Lemma 3.3), we can rewrite the sum over $r$ in (6.5) as the action of the free operators:

$$
\prod_{j=1}^{b}\left[\nabla_{v_{n} / v_{n+1}}^{\mathrm{beta}}\right]_{\ell+a+j} g(t ; m_{1}, \ldots, m_{\ell}, \underbrace{n+1, \ldots, n+1}_{a+b}, m_{1}^{\prime}, \ldots, m_{\ell^{\prime}}^{\prime}) .
$$

Finally, each of the operators $\nabla_{v_{n} / v_{n+1}}^{\text {beta }}$ can be applied separately under the contour integral in $g(t ; \cdot)$ given by $(6.1)$, and we obtain (with the notation $w=z_{\ell+a+j}, j=1, \ldots, b$ ):

$$
\begin{aligned}
{\left[\nabla_{v_{n} / v_{n+1}}^{\text {beta }}\right]_{\ell+a+j} \prod_{i=1}^{n+1} \frac{w}{w-v_{i}} } & =\left(\frac{v_{n}}{v_{n+1}}+\left(1-\frac{v_{n}}{v_{n+1}}\right) \frac{w}{w-v_{n+1}}\right) \prod_{i=1}^{n} \frac{w}{w-v_{i}} \\
& =\frac{w-v_{n}}{w-v_{n+1}} \prod_{i=1}^{n} \frac{w}{w-v_{i}}=\prod_{\substack{i=1 \\
i \neq n}}^{n+1} \frac{w}{w-v_{i}}
\end{aligned}
$$

Thus, we see that (6.4) is equal to the expectation $\mathbb{E}^{\left.\text {beta( } s_{n} \boldsymbol{v}\right)}\left(Z\left(t, n_{1}\right) \cdots Z\left(t, n_{k}\right)\right)$ with the swapped parameters $v_{n} \leftrightarrow v_{n+1}$, where $\left(n_{1}, \ldots, n_{k}\right)$ is given by (6.3). Since joint moments determine the distribution of the beta polymer, we are done.

\subsection{Polymer interpretation}

Let us give a polymer interpretation of Theorem 6.4 (assuming that $v_{n}<v_{n+1}$ ). First, observe that the quantity

$$
\tilde{Z}(t, n):=\tilde{B}_{n} Z(t, n+1)+\left(1-\tilde{B}_{n}\right) Z(t, n),
$$

where $\tilde{B}_{n} \sim \operatorname{Beta}\left(\vee_{n+1}-v_{n}, v_{n}\right)$, is a beta polymer type partition function on a modified lattice. This modified lattice coincides with the one in Fig. 9 in the vertical strip $\{0,1, \ldots, t\} \times \mathbb{Z}_{\geq 1}$, has an additional vertex $\mathrm{A}$, and two additional directed edges $(t, n) \rightarrow \mathrm{A}$ and $(t, n+1) \rightarrow \mathrm{A}$ with weights $1-\tilde{B}_{n}$ and $\tilde{B}_{n}$, respectively. The partition function from the line $\{0\} \times \mathbb{Z}_{\geq 1}$ to $\mathrm{A}$ is precisely $\tilde{Z}(t, n)$. See Fig. 10 for an illustration.

Let us now iterate the swapping in Theorem 6.4 and interchange the parameter $v_{1}$ with $v_{2}$, then with $v_{3}$, and so on up to infinity. Assume that $v_{1}<v_{2}<\cdots$. Let $B_{n}^{(1)} \sim \operatorname{Beta}\left(v_{n+1}-\right.$ $\left.v_{1}, v_{1}\right), n \in \mathbb{Z}_{\geq 1}$, be independent random variables which are also independent of the environment $\left\{B_{t, n}\right\}$ in the beta polymer. Define

$$
Z^{(1)}(t, n):=B_{n}^{(1)} Z(t, n+1)+\left(1-B_{n}^{(1)}\right) Z(t, n), \quad n=1,2, \ldots
$$

Proposition 6.5. The joint distribution of $\left\{Z^{(1)}(t, n)\right\}_{n \in \mathbb{Z}_{\geq 1}}$ defined above coincides with the joint distribution of the beta polymer $\left\{Z^{\left(v_{2}, v_{3}, \ldots\right)}(t, n)\right\}_{n \in \mathbb{Z}_{\geq 1}}$ at same $t$, but with the sequence of parameters shifted by one. 


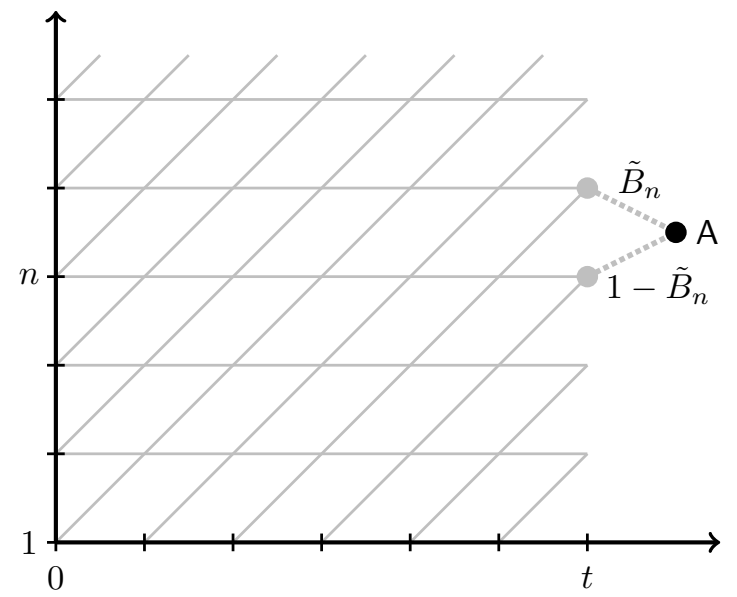

Figure 10. Modified lattice of finite width used to interpret $\tilde{Z}(t, n)(6.6)$ as a polymer partition function.

Proof. Fix $m \in \mathbb{Z}_{\geq 1}$. Observe that the joint distribution of $\left(Z^{\left(v_{1}, v_{2}, \ldots\right)}(t, m), Z^{\left(v_{1}, v_{2}, \ldots\right)}(t, m+1)\right)$ does not depend on the order of the parameters $v_{1}, \ldots, v_{m}$. Therefore, applying (6.7) with $n=m$ and using Theorem 6.4 makes the new random variable $Z^{(1)}(t, m)$ a beta polymer partition function with parameters $\left(v_{2}, \ldots, v_{n}, v_{n+1}\right)$. The statement about joint distributions is obtained by sequential application of this argument for $m=1,2, \ldots$.

The quantities $\left\{Z^{(1)}(t, n)\right\}_{n \in \mathbb{Z}_{\geq 1}}$ can be interpreted as beta polymer type partition functions, too. Moreover, let us further iterate Proposition 6.5, and introduce independent random variables

$$
B_{n}^{(s)} \sim \operatorname{Beta}\left(v_{n+s}-v_{s}, v_{s}\right), \quad s=1,2, \ldots
$$

Define $Z^{(s)}(t, n)$ to be the polymer partition function from the line $\{0\} \times \mathbb{Z}_{\geq 1}$ to the point $(s+t, n)$, $s \in \mathbb{Z}_{\geq 1}$, in the modified strict-weak lattice which coincides with the original lattice in Fig. 9 in the vertical strip $\{0,1, \ldots, t\} \times \mathbb{Z}_{\geq 1}$. To the right of this strip, the modified lattice is made out of down-right diagonal and horizontal edges with the weights $B_{n}^{(s)}$ on each $(t+s-1, n+1) \rightarrow$ $(t+s, n)$, and $1-B_{n}^{(s)}$ on each $(t+s-1, n) \rightarrow(t+s, n)$. See Fig. 11 for an illustration.

Proposition 6.6. For any fixed $s$ and $t$, the joint distribution of the partition functions $\left\{Z^{(s)}(t, n)\right\}_{n \in \mathbb{Z}_{>1}}$ on the modified lattice coincides with the joint distribution of the beta polymer partition functions $\left\{Z^{\left(v_{s+1}, v_{s+2}, \ldots\right)}(t, n)\right\}_{n \in \mathbb{Z}_{\geq 1}}$ with the same $t$, but with the parameter sequence $\boldsymbol{v}$ shifted by $s$.

\subsection{Zero-temperature limit}

Under a limit transition when the parameters of the beta random variables go to zero, the beta polymer model turns into a first passage percolation type model. First, we recall the scaling:

Lemma 6.7 ([4, Lemma 5.1]). Let $\alpha, \beta>0$, and $B_{\varepsilon} \sim \operatorname{Beta}(\varepsilon \alpha, \varepsilon \beta)$. Then, as $\varepsilon \searrow 0$, we have convergence in distribution:

$$
\left(-\varepsilon \log B_{\varepsilon},-\varepsilon \log \left(1-B_{\varepsilon}\right)\right) \rightarrow\left(\xi E_{\alpha},(1-\xi) E_{\beta}\right) .
$$

Here $\xi \in\{0,1\}$ is the Bernoulli random variable with $\mathbb{P}(\xi=1)=\frac{\beta}{\alpha+\beta}$, and $\left(E_{\alpha}, E_{\beta}\right)$ are exponential random variables with parameters $\alpha$ and $\beta$ (that is, means $\alpha^{-1}$ and $\beta^{-1}$ ) which are independent of $\xi$. 


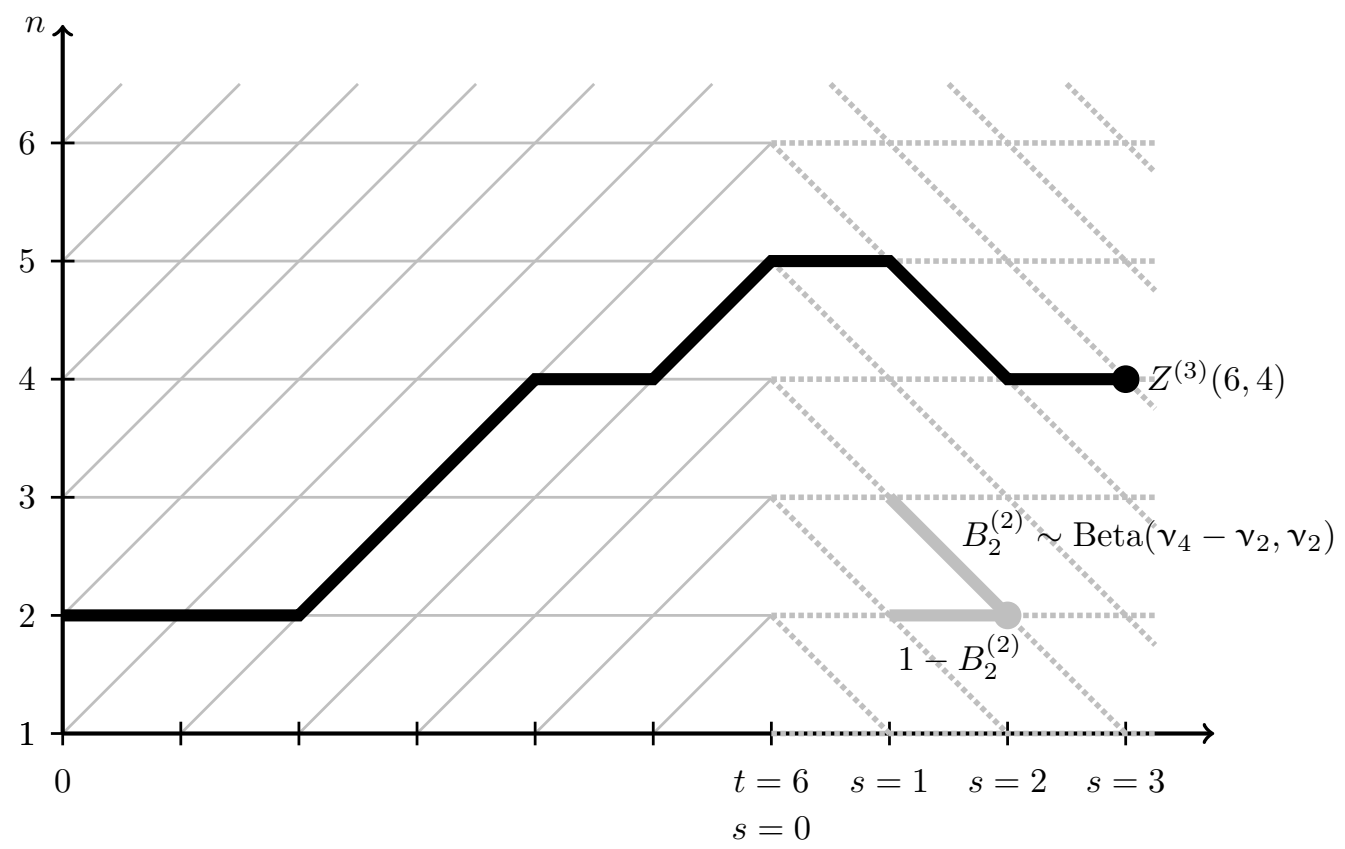

Figure 11. The lattice used to define the beta polymer partition functions $Z^{(s)}(t, n)$ with shifted parameter sequences.

We will take the scaling limit of the beta polymer model as $v_{n}=\varepsilon \overline{\boldsymbol{v}}_{n}, \gamma=\varepsilon \bar{\gamma}$, where $\bar{v}_{n}>\bar{\gamma}>0$ for all $n$, and $0<\bar{v}_{1}<\bar{v}_{2}<\cdots$. The edge weights in the lattice in Fig. 11 turn into the ones given in Fig. 12.
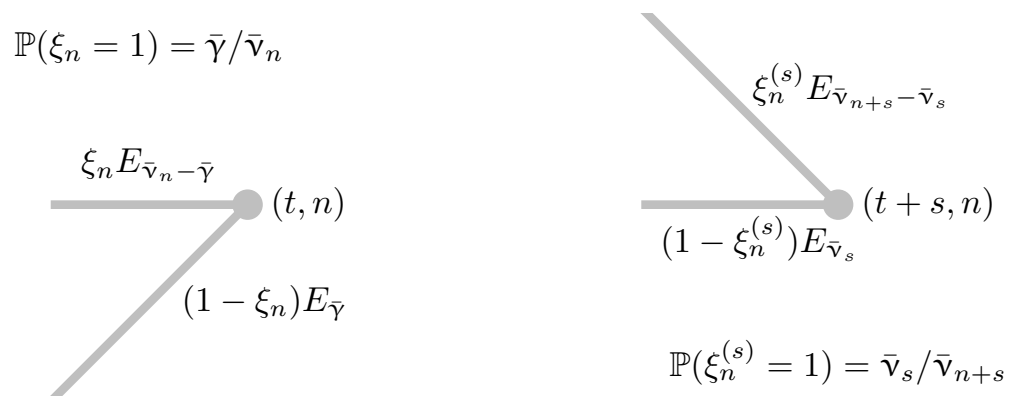

Figure 12. Edge weights $t_{e}$ in the zero-temperature limit. The $\xi$ 's are independent Bernoulli random variables with given parameters, and all the $E_{\alpha}$ 's are exponential random variables independent of the Bernoulli ones.

Denote by $F^{(s)}(t, n)$ the first-passage time from the line $\{0\} \times \mathbb{Z}_{\geq 1}$ to the point $(s+t, n)$ in the modified lattice

$$
F^{(s)}(t, n):=\min _{\pi:\{0\} \times \mathbb{Z}_{\geq 1} \rightarrow(t+s, n)} \sum_{e \in \pi} t_{e},
$$

where the directed paths $\pi$ are as in Fig. 11, and the edge weights are given in Fig. 12. If $s=0$, then we mean the unmodified first-passage time (as studied in [4]). Above the main diagonal (i.e., for $n>t$ ) we have $F^{(0)}(t, n)=0$ because due to the presence of the Bernoulli components, there always exists a path with zero total weight between $(t, n), n>t$, and the vertical axis $\{0\} \times \mathbb{Z}_{\geq 1}$.

For the first-passage percolation model, an analogue of Proposition 6.6 holds: 
Proposition 6.8. For fixed $s, t$, the joint distribution of the first-passage times $\left\{F^{(s)}(t, n)\right\}_{n \in \mathbb{Z}_{\geq 1}}$ with parameters $\bar{v}_{1}<\bar{v}_{2}<\cdots$ in the modified lattice is the same as that of the unmodified ones $\left\{F^{(0)}(t, n)\right\}_{n \in \mathbb{Z}_{\geq 1}}$, but with the shifted sequence of parameters $\bar{v}_{s+1}<\bar{v}_{s+2}<\cdots$.

\section{Acknowledgements}

I am grateful to Vadim Gorin for helpful discussions, and to Matteo Mucciconi and Axel Saenz for remarks on the first version of the manuscript. I am grateful to the organizers of the workshop "Dimers, Ising Model, and their Interactions" and the support of the Banff International Research Station where a part of this work was done. The work was partially supported by the NSF grant DMS-1664617.

\section{References}

[1] Assiotis T., Determinantal structures in space-inhomogeneous dynamics on interlacing arrays, Ann. Henri Poincaré 21 (2020), 909-940, arXiv:1910.09500.

[2] Barraquand G., A short proof of a symmetry identity for the $q$-Hahn distribution, Electron. Commun. Probab. 19 (2014), no. 50, 3 pages, arXiv:1404.4265.

[3] Barraquand G., Corwin I., The $q$-Hahn asymmetric exclusion process, Ann. Appl. Probab. 26 (2016), 23042356, arXiv:1501.03445.

[4] Barraquand G., Corwin I., Random-walk in beta-distributed random environment, Probab. Theory Related Fields 167 (2017), 1057-1116, arXiv:1503.04117.

[5] Basu R., Sarkar S., Sly A., Last passage percolation with a defect line and the solution of the slow bond problem, arXiv:1408.3464.

[6] Basu R., Sarkar S., Sly A., Invariant measures for TASEP with a slow bond, arXiv:1704.07799.

[7] Borodin A., Corwin I., Macdonald processes, Probab. Theory Related Fields 158 (2014), 225-400, arXiv:1111.4408.

[8] Borodin A., Corwin I., Gorin V., Stochastic six-vertex model, Duke Math. J. 165 (2016), 563-624, arXiv:1407.6729.

[9] Borodin A., Corwin I., Petrov L., Sasamoto T., Spectral theory for interacting particle systems solvable by coordinate Bethe ansatz, Comm. Math. Phys. 339 (2015), 1167-1245, arXiv:1407.8534.

[10] Borodin A., Corwin I., Sasamoto T., From duality to determinants for $q$-TASEP and ASEP, Ann. Probab. 42 (2014), 2314-2382, arXiv:1207.5035.

[11] Borodin A., Ferrari P.L., Anisotropic growth of random surfaces in $2+1$ dimensions, Comm. Math. Phys. 325 (2014), 603-684, arXiv:0804.3035.

[12] Borodin A., Gorin V., Wheeler M., Shift-invariance for vertex models and polymers, arXiv:1912.02957.

[13] Borodin A., Petrov L., Integrable probability: stochastic vertex models and symmetric functions, in Stochastic Processes and Random Matrices, Oxford University Press, Oxford, 2017, 26-131, arXiv:1605.01349.

[14] Borodin A., Petrov L., Higher spin six vertex model and symmetric rational functions, Selecta Math. (N.S.) 24 (2018), 751-874, arXiv:1601.05770.

[15] Borodin A., Petrov L., Inhomogeneous exponential jump model, Probab. Theory Related Fields 172 (2018), 323-385, arXiv:1703.03857.

[16] Borodin A., Wheeler M., Coloured stochastic vertex models and their spectral theory, arXiv:1808.01866.

[17] Bufetov A., Mucciconi M., Petrov L., Yang-Baxter random fields and stochastic vertex models, Adv. Math., to appear, arXiv:1905.06815.

[18] Corwin I., Invariance of polymer partition functions under the geometric RSK correspondence, arXiv:2001.01867.

[19] Corwin I., The Kardar-Parisi-Zhang equation and universality class, Random Matrices Theory Appl. 1 (2012), 1130001, 76 pages, arXiv:1106.1596.

[20] Corwin I., The $q$-Hahn boson process and $q$-Hahn TASEP, Int. Math. Res. Not. 2015 (2015), 5577-5603, arXiv:1401.3321. 
[21] Corwin I., Kardar-Parisi-Zhang universality, Notices Amer. Math. Soc. 63 (2016), 230-239.

[22] Corwin I., Matveev K., Petrov L., The q-Hahn PushTASEP, Int. Math. Res. Not. 2021 (2021), 2210-2249, arXiv:1811.06475.

[23] Corwin I., O'Connell N., Seppäläinen T., Zygouras N., Tropical combinatorics and Whittaker functions, Duke Math. J. 163 (2014), 513-563, arXiv:1110.3489.

[24] Corwin I., Petrov L., Stochastic higher spin vertex models on the line, Comm. Math. Phys. 343 (2016), 651-700, arXiv:1502.07374.

[25] Costin O., Lebowitz J.L., Speer E.R., Troiani A., The blockage problem, Bull. Inst. Math. Acad. Sin. (N.S.) 8 (2013), 49-72, arXiv:1207.6555.

[26] Dauvergne D., Hidden invariance of last passage percolation and directed polymers, arXiv:2002.09459.

[27] Ferrari P.L., Vető B., Tracy-Widom asymptotics for q-TASEP, Ann. Inst. Henri Poincaré Probab. Stat. 51 (2015), 1465-1485, arXiv:1310.2515.

[28] Galashin P., Symmetries of stochastic colored vertex models, arXiv:2003.06330.

[29] Gravner J., Tracy C.A., Widom H., Fluctuations in the composite regime of a disordered growth model, Comm. Math. Phys. 229 (2002), 433-458, arXiv:math.PR/0111036.

[30] Gwa L.H., Spohn H., Six-vertex model, roughened surfaces, and an asymmetric spin Hamiltonian, Phys. Rev. Lett. 68 (1992), 725-728.

[31] Halpin-Healy T., Takeuchi K.A., A KPZ cocktail - shaken, not stirred ... toasting 30 years of kinetically roughened surfaces, J. Stat. Phys. 160 (2015), 794-814, arXiv:1505.01910.

[32] Its A.R., Tracy C.A., Widom H., Random words, Toeplitz determinants and integrable systems. II, Phys. D 152/153 (2001), 199-224, arXiv:nlin.SI/0004018.

[33] Janowsky S.A., Lebowitz J.L., Finite-size effects and shock fluctuations in the asymmetric simple-exclusion process, Phys. Rev. A 45 (1992), 618-625.

[34] Johansson K., Shape fluctuations and random matrices, Comm. Math. Phys. 209 (2000), 437-476, arXiv:math.CO/9903134.

[35] Knizel A., Petrov L., Saenz A., Generalizations of TASEP in discrete and continuous inhomogeneous space, Comm. Math. Phys. 372 (2019), 797-864, arXiv:1808.09855.

[36] Mucciconi M., Petrov L., Spin $q$-Whittaker polynomials and deformed quantum Toda, arXiv:2003.14260

[37] O'Connell N., Directed polymers and the quantum Toda lattice, Ann. Probab. 40 (2012), 437-458, arXiv:0910.0069.

[38] O'Connell N., Seppäläinen T., Zygouras N., Geometric RSK correspondence, Whittaker functions and symmetrized random polymers, Invent. Math. 197 (2014), 361-416, arXiv:1110.3489.

[39] Okounkov A., Reshetikhin N., Correlation function of Schur process with application to local geometry of a random 3-dimensional Young diagram, J. Amer. Math. Soc. 16 (2003), 581-603, arXiv:math.CO/0107056.

[40] Petrov L., PushTASEP in inhomogeneous space, Electron. J. Probab. 25 (2020), 114, 25 pages, arXiv:1910.08994.

[41] Petrov L., Saenz A., Mapping TASEP back in time, arXiv:1907.09155.

[42] Povolotsky A.M., On the integrability of zero-range chipping models with factorized steady states, J. Phys. A: Math. Theor. 46 (2013), 465205, 25 pages, arXiv:1308.3250.

[43] Quastel J., Spohn H., The one-dimensional KPZ equation and its universality class, J. Stat. Phys. 160 (2015), 965-984, arXiv:1503.06185.

[44] Rákos A., Schütz G.M., Bethe ansatz and current distribution for the TASEP with particle-dependent hopping rates, Markov Process. Related Fields 12 (2006), 323-334, arXiv:cond-mat/0506525.

[45] Sasamoto T., Wadati M., Exact results for one-dimensional totally asymmetric diffusion models, J. Phys. A: Math. Gen. 31 (1998), 6057-6071.

[46] Seppäläinen T., Hydrodynamic profiles for the totally asymmetric exclusion process with a slow bond, J. Stat. Phys. 102 (2001), 69-96, arXiv:math.PR/0003049.

[47] Seppäläinen T., Scaling for a one-dimensional directed polymer with boundary conditions, Ann. Probab. 40 (2012), 19-73, arXiv:0911.2446.

[48] Takeyama Y., A deformation of affine Hecke algebra and integrable stochastic particle system, J. Phys. A: Math. Theor. 47 (2014), 465203, 19 pages, arXiv:1407.1960. 
[49] Thiery T., Le Doussal P., On integrable directed polymer models on the square lattice, J. Phys. A: Math. Theor. 48 (2015), 465001, 41 pages, arXiv:1506.05006.

[50] Tracy C.A., Widom H., Integral formulas for the asymmetric simple exclusion process, Comm. Math. Phys. 279 (2008), 815-844, Erratum, Comm. Math. Phys. 304 (2011), 875-878, arXiv:0704.2633.

[51] Tracy C.A., Widom H., Asymptotics in ASEP with step initial condition, Comm. Math. Phys. 290 (2009), 129-154, arXiv:0807.1713.

[52] Vető B., Tracy-Widom limit of q-Hahn TASEP, Electron. J. Probab. 20 (2015), 102, 22 pages, arXiv:1407.2787. 